\title{
CLASSIC AND EXOTIC BESOV SPACES INDUCED BY GOOD GRIDS
}

\author{
DANIEL SMANIA
}

\begin{abstract}
In a previous work we introduced Besov spaces $\mathcal{B}_{p, q}^{s}$ defined on a measure spaces with a good grid, with $p \in[1, \infty), q \in[1, \infty]$ and $0<s<1 / p$. Here we show that classical Besov spaces on compact homogeneous spaces are examples of such Besov spaces. On the other hand we show that even Besov spaces defined by a good grid made of partitions by intervals may differ from a classical Besov space, giving birth to exotic Besov spaces.
\end{abstract}

\section{Contents}

1. Introduction

1.1. Measure spaces with good grids

1.2. Besov space on measure spaces with good grid

1.3. Comparing Besov spaces

2

3

I. THE BEST OF ALL POSSIBLE WORLDS.

2. Besov spaces on compact homogeneous spaces

2.1. Homogeneous spaces

2.2. Induced Ahlfors regular quasi-metric space.

2.3. Good grids in an Ahlfors regular quasi-metric space. 5

2.4. The case $[0,1]^{D}$. 14

3. Gu-Taibleson recalibrated martingale Besov spaces 14

$\begin{array}{ll}\text { 4. Examples of strongly regular domains } & 17\end{array}$

II. GETTING $\mathbb{R E A L . ~} \quad 20$

5. Isometry with Besov spaces defined by intervals 20

6. Quasisymmetric grids 20

7. Exotic $\mathcal{B}_{p, q}^{s}$ for $p \neq q$

8. Good grid invariance for $\mathcal{B}_{p, p}^{s} \quad 31$

References 33

2010 Mathematics Subject Classification. 37C30, 30H25, 42B35, 42C15, 42C40.

Key words and phrases. atomic decomposition, Besov space, harmonic analysis, wavelets.

D.S. was partially supported by CNPq 307617/2016-5, CNPq 430351/2018-6, CNPq 306622/2019-0 and FAPESP Projeto Temático 2017/06463-3. 


\section{INTRODUCTION}

We defined Besov spaces $\mathcal{B}_{p, q}^{s}$, with $p \in[1, \infty), 0<s<1 / p$ and $q \in[1, \infty]$, on a measure spaces with a good grid [27]. Those spaces are defined by atomic decomposition using very simple atoms consisting of piecewise constant functions.

Similar but more general than Gu-Taibleson [14] recalibrated martingale Besov spaces, it allows us to carry results for classic Besov spaces in $\mathbb{R}^{n}$ to measure spaces with a good grid, and often the proofs there are indeed simpler and more elementary. This includes results on multipliers, atomic decomposition and left and right compositions.

1.1. Measure spaces with good grids. A measure space with a good grid is a set $I$ endowed with a $\sigma$-algebra $\mathbb{A}$ and a measure $m$ on $(I, \mathbb{A}), m(I)<\infty$. For every measurable set $S$ denote $|S|=m(S)$. A good grid on $I$ is a sequence of finite families of measurable sets with positive measure $\mathcal{P}=\left(\mathcal{P}^{k}\right)_{k \in \mathbb{N}}$ so that

i. Every family $\mathcal{P}^{k}$ is a partition of $I$ up to sets of zero measure.

ii. The family

$$
\cup_{k} \mathcal{P}^{k}
$$

generates the $\sigma$-algebra $\mathbb{A}$.

iii. There is $\lambda, \hat{\lambda} \in(0,1)$ such that

$$
\hat{\lambda} \leq \frac{|Q|}{|P|} \leq \lambda
$$

for all $Q \subset P$ such that $Q \in \mathcal{P}^{k+1}$ and $P \in \mathcal{P}^{k}$ for some $k \geq 0$.

1.2. Besov space on measure spaces with good grid. For each $Q \in \mathcal{P}$ consider the function $a_{Q}$ defined by

$$
a_{Q}(x)=|Q|^{s-1 / p}
$$

for every $x \in Q$ and $a_{Q}(x)=0$ otherwise. The function $a_{Q}$ is the Souza's canonical atom on $Q$. The Besov space $\mathcal{B}_{p, q}^{s}$ is the space of all functions $f$ in the Lebesgue space $L^{p}$ that can be represented by an absolutely convergent series on $L^{p}$

$$
f=\sum_{k=0}^{\infty} \sum_{Q \in \mathcal{P}^{k}} s_{Q} a_{Q}
$$

where $s_{Q} \in \mathbb{C}$ and additionally

$$
\left(\sum_{k=0}^{\infty}\left(\sum_{Q \in \mathcal{P}^{k}}\left|s_{Q}\right|^{p}\right)^{q / p}\right)^{1 / q}<\infty .
$$

The r.h.s. of (1.1) is a $\mathcal{B}_{p, q}^{s}$-representation of $f$. Define

$$
|f|_{\mathcal{B}_{p, q}^{s}}=\inf \left(\sum_{k=0}^{\infty}\left(\sum_{Q \in \mathcal{P}_{k}}\left|s_{Q}\right|^{p}\right)^{q / p}\right)^{1 / q},
$$

where the infimum runs over all possible representations of $f$ as in (1.1). Then $\left(\mathcal{B}_{p, q}^{s},|\cdot|_{\mathcal{B}_{p, q}^{s}}\right)$ is a complex Banach space and its unit ball is compact in $L^{p}$ (see $[27])$. 
1.3. Comparing Besov spaces. There is a large body of literature on Besov spaces. See Stein [28], Peetre [24], Triebel [31] and the references therein. Since Besov [4] defined Besov spaces $\mathbb{R}^{n}$ in late 50's, there is a long and ongoing quest to extend Besov spaces (and indeed harmonic analysis) to settings with weaker structure. Han and Sawyer [16] and Han, Lu and Yang [15] defined Besov spaces on homogeneous spaces. Those are a large class of quasi-metric spaces endowed with a doubling measure, introduced by Coifman and Weiss [8]. There are also definitions of Besov spaces on metric spaces and $d$-sets. See Alvarado and Mitrea [2] and Koskela, Yang, and Zhou [19] and Triebel [32][30].

Our goal here is to compare the Besov spaces of a homogeneous space with the ones defined on a measure spaces with a good grid. While the former is certainly a far-reaching generalisation of Besov spaces on $\mathbb{R}^{n}$, the latter may at first glance looks like an artificial dyadic version of the classical Besov spaces, a sort of simplistic model of more complex situations. As it turn out, this couldnt be farther from the truth.

Indeed there are earlier works that give atomic decompositions to classical Besov spaces where the atoms are piecewise constant functions. We cite the atomic decomposition of the Besov space $B_{1,1}^{s}([a, b])$, with $s \in(0,1)$, by de Souza [9] (see De Souza [10] and de Souza, O'Neil and Sampson [11]), some results on B-spline atomic decomposition of the Besov space of the unit cube of $\mathbb{R}^{n}$ by DeVore and Popov [12], as well results on finite element approximation in bounded polyhedral domains on $\mathbb{R}^{n}$ by Oswald [21] [22].

In part I, we consider compact homogeneous spaces, and using the famous dyadic "cubes" constructed by Christ [7] (see also Hytönen and Kairema [17] for recent results on dyadic cubes in homogeneous spaces), we show that its Besov spaces, as defined by Han, Lu and Yang [15], are a particular case of the Besov spaces induced by grids. Note that Yang [35] already proved that Besov spaces on $d$-sets defined by Triebel [32] also coincide with Besov spaces on these sets considering them as homogeneous spaces. We also observe that $\mathrm{Gu}$-Taibleson recalibrated martingale Besov spaces [14] are Besov spaces of certain compact homogeneous spaces (see Section 3).

Besov spaces on compact homogeneous spaces, with $p \in[1, \infty), q \geq 1$ and small $s$ satisfying $0<s<1 / p$, and in particular recalibrated martingale Besov spaces, can be retrieved as particular examples of Besov spaces in measure spaces with a good grid.

On the other hand in Part II we show that, even if we consider $[0,1]$ with a grid formed by a nested sequence of partitions by intervals, but that it is not "recalibrated" as in Gu and Taibleson [14], then it may happens that the induced Besov space does not coincide with the Besov space of $[0,1]$. Those "exotic" Besov spaces are indeed useful to study certain transfer operators associated to expanding maps [3].

Yet our construction of Besov spaces $B_{p, q}^{s}$ with low regularity $s$ is likely to be the simplest construction available. 


\section{THE BEST OF ALL POSSIBLE WORLDS.}

\section{BESOV SPACES ON COMPACT HOMOGENEOUS SPACES}

2.1. Homogeneous spaces. Let $I$ be a set. A quasi-metric $\rho(\cdot, \cdot)$ in $I$ is a realvalued function in $I \times I$ satisfying

HS1. We have $\rho(x, x)=0$,

HS2. If $x \neq y$ then $\rho(x, y)>0$,

HS3. We have $\rho(x, y)=\rho(y, x)$,

HS4. There exists $C_{1} \geq 1$ such that

$$
\rho(x, z) \leq C_{1}(\rho(x, y)+\rho(y, z))
$$

For every $\gamma>0$ the function $\rho^{\gamma}$ is also a quasi-metric on $I$. A quasi-metric $\rho^{\prime}$ is equivalent to $\rho$ if

$$
\frac{1}{C} \rho^{\prime}(x, y) \leq \rho(x, y) \leq C \rho^{\prime}(x, y)
$$

for some $C \geq 1$. We say that two quasi-metrics $\rho$ and $\rho^{\prime}$ are power-law equivalent if there exists $\gamma>0$ such that $\rho^{\prime}$ is equivalent to $\rho^{\gamma}$.

A homogeneous space $(I, \rho, m)$, introduced by Coifman and Weiss [8], is a topological space $I$ endowed with a quasi-metric $\rho(\cdot, \cdot)$ and a borelian measure $m$ that satisfies

HS5. The measure $m$ is a doubling measure, that is, there is $C>0$ such that

$$
0<m\left(B_{\rho}(x, r)\right)<C m\left(B_{\rho}(x, r / 2)\right)<\infty .
$$

for every $x \in I$ and $r>0$.

Note that if $\rho^{\prime}$ is power-law equivalent to $\rho$ then $\left(I, \rho^{\prime}, m\right)$ is also a homogeneous space. By Macias and Segovia [20], replacing $\rho$ by an equivalent quasi-metric we can also assume

HS6. The quasi-balls $B_{\rho}(x, r)$, with $r>0$, are open sets.

We will assume that $m(\{x\})=0$ for every $x \in I, I$ is compact and $m(I)=1$.

2.2. Induced Ahlfors regular quasi-metric space. For every homogeneous space as in the previous section we can associated an Ahlfors regular quasi-metric space, that is, a triple $(I, m, d, D)$, where $d$ is a quasi-metric, $D>0$ and

$$
\frac{1}{C_{2}} r^{D} \leq m\left(B_{d}(x, r)\right) \leq C_{2} r^{D}
$$

provided $r \leq r_{0}$, for some $C_{2}, r_{0}>0$. Indeed $d$ is defined up to power law equivalence, but that will be enough to ours purposes.

Indeed by Macias and Segovia [20] if we define

$$
\beta(x, y)=\inf \{m(B): B \text { is a } \rho \text {-ball that contains }\{x, y\}\}
$$

then $\beta$ is a quasi-metric on $I$ (that it is not necessarily equivalent to $\rho$ ) such that $(I, \beta, m)$ is a homogeneous space satisfying

$$
\frac{1}{C_{3}} r \leq m\left(B_{\beta}(x, r)\right) \leq C_{3} r
$$


provided $r \leq r_{0}$, for some $C_{3}, r_{0}>0$, so $(m, \beta, 1)$ is a Ahlfors regular quasi-metric space. Note that if $\rho$ and $\rho^{\prime}$ are power-law equivalent then the corresponding quasimetrics $\beta$ and $\beta^{\prime}$ are equivalent.

One may ask if there is a metric $d$ and $D>0$ such that $(m, d, D)$ is an Ahlfors regular metric space and $d$ is power-law equivalent to $\beta$. Indeed given $\gamma>0$ such that $\left(2 C_{1}\right)^{\gamma} \leq 2$ we have that $d_{\gamma}$ is a metric, where

$$
d_{\gamma}(x, y)=\inf \left\{\sum_{i=1}^{m-1} \beta\left(x_{i}, x_{i+1}\right)^{\gamma}, x_{i} \in I, x_{1}=x \text { and } x_{m}=y\right\} .
$$

By Aimar, Iaffei and Nitti [1] and Paluszyński and Stempak [23] if $\left(2 C_{1}\right)^{\gamma} \leq 2$ then $d_{\gamma}$ is a metric on $I, d_{\gamma}^{1 / \gamma}$ is a quasi-metric equivalent to $\beta$. In particular $\beta, d_{\gamma}$ and $d_{\gamma^{\prime}}$ are power-law equivalent for every $\gamma, \gamma^{\prime}$. Moreover

$$
\frac{1}{C_{4}} r^{1 / \gamma} \leq m\left(B_{d_{\gamma}}(x, r)\right) \leq C_{4} r^{1 / \gamma}
$$

for some $C_{4}>1$ and $r \leq r_{2}$. So $\left(m, d_{\gamma}, 1 / \gamma\right)$ is a Ahlfors regular metric space.

2.3. Good grids in an Ahlfors regular quasi-metric space. Due Section 2.2 from now on we will consider a general setting of Ahlfors regular quasi-metric space $(m, d, D)$ satisfying (2.4) for $r \leq r_{0}$ and some $C_{2}, r_{0}>0$.

Proposition 2.1 (Good grids in Ahlfors regular quasi-metric spaces). There is a good grid $\mathcal{P}=\left(\mathcal{P}^{i}\right)_{i}$ such that for every quasi-metric $\alpha$ that is power-law equivalent to $d$ the following holds: There is $\eta, C_{5}, C_{6}, C_{7}, C_{8} \geq 0$ and $\lambda_{1} \in(0,1)$ such that for every $Q \in \mathcal{P}^{k}$, with $k \geq 1$, there is $z_{Q} \in Q$ satisfying

$$
\begin{gathered}
B_{\alpha}\left(z_{Q}, C_{5} \lambda_{1}^{k}\right) \subset Q, \\
\operatorname{diam}_{\alpha} Q \leq C_{6} \lambda_{1}^{k}
\end{gathered}
$$

and

$$
m\left\{x \in Q: \alpha(x, I \backslash Q) \leq C_{7} t \lambda_{1}^{k}\right\} \leq C_{8} t^{\eta} m(Q) .
$$

Proof. By Christ [7] there is a family of partitions $\mathcal{G}^{k}, k \in \mathbb{Z}$, satisfying

C1. For every $k$ we have $m\left(I \backslash \cup_{Q \in \mathcal{G}^{k}} Q\right)=0$.

C2. For every $Q, W \in \mathcal{G}^{k}$ we have either $Q=W$ or $Q \cap W=\emptyset$.

C3. For every $Q \in \mathcal{G}^{k}$ there is an unique $P \in \mathcal{P}^{k-1}$ such that $Q \subset P$.

C4. For every $Q \in \mathcal{G}^{k}$ we have $\operatorname{diam}_{d} Q \leq C_{9} \lambda_{2}{ }^{k}$.

C5. For every $Q \in \mathcal{G}^{k}$ there is $z_{Q} \in Q$ such that $B_{d}\left(z_{Q}, C_{10} \lambda_{2}^{k}\right) \subset Q$, with $C_{10} \leq C_{9}$.

C6. For every $Q \in \mathcal{G}^{k}$ we have

$$
m\left\{x \in Q: d(x, I \backslash Q) \leq t \lambda_{2}^{k}\right\} \leq C_{8} t^{\eta} m(Q) .
$$

Let $k_{0}$ be such that

$$
\left(C_{10}+C_{9}\right) \lambda_{2}^{k_{0}}<r_{1}
$$

and

Let $K \in \mathbb{N}$ be such that

$$
C_{2} C_{9} \lambda_{2}^{k_{0}}<1
$$

$$
\lambda_{6}=\max \left\{C_{2}^{2} \frac{C_{9}^{D}}{C_{10}^{D}} \lambda_{2}^{D K}, C_{2} C_{9}^{D} \lambda_{2}^{D k_{0}}\right\}<1 .
$$


Note that

$$
\lambda_{5}=\min \left\{\frac{1}{C_{2}^{2}} \frac{C_{10}^{D}}{C_{9}^{D}} \lambda_{2}^{D K}, \frac{1}{C_{2}} C_{10}^{D} \lambda_{2}^{D k_{0}}\right\} \leq \lambda_{6} .
$$

Let $\mathcal{P}^{0}=\{I\}$ and $\mathcal{P}^{i}=\mathcal{G}^{k_{0}+(i-1) K}$ for every $i \geq 1$. Then for every $i \geq 0, P \in \mathcal{P}^{i+1}$ and $P \subset Q \in \mathcal{P}^{i}$ we have

$$
\lambda_{5} \leq \frac{|P|}{|Q|} \leq \lambda_{6}
$$

It is easy to see that $\mathcal{P}$ has similar properties for every power-law equivalent metric $\alpha$.

For every grid $\mathcal{P}$ as in Proposition 2.1 we can consider the Banach space $\mathcal{B}_{p, q}^{s}(\mathcal{P})$, using $(s, p)$-Souza's atoms, where $p \in[1, \infty), q \in[1, \infty]$ and $s \in(0,1 / p)$.

Proposition 2.2. Let $\mathcal{P}$ be a grid as in Proposition 2.1 taking $\alpha=d$. Assume that $D s p<\hat{\eta}$. There is $C_{11}, C_{12}$ such that the following holds. Let $Q \subset I$ be an open subset such that there is $z_{Q} \in Q$ satisfying

$$
B_{d}\left(z_{Q}, C_{13} \operatorname{diam}_{d} Q\right) \subset Q,
$$

and

$$
m\left\{x \in Q: d(x, I \backslash Q) \leq C_{14} t \operatorname{diam}_{d} Q\right\} \leq C_{15} t^{\hat{\eta}} m(Q)
$$

for some $C_{13}, C_{14}, C_{15}$ and $\hat{\eta}>0$. Then

A. The set $Q$ is a $\left(1-s p, C_{16}, \lambda_{1}^{\hat{\eta}-D s p}\right)$-regular domain, with

$$
C_{16}=C_{11} \frac{C_{15}}{C_{14}^{\hat{\gamma}} C_{13}^{D}}
$$

that is, there are families $\mathcal{F}_{Q}^{k} \subset \mathcal{P}^{k}$, such that

i. If $P \in \mathcal{F}_{Q}^{k}, W \in \mathcal{F}_{Q}^{j}$ and $P \neq W$ then $P \cap W=\emptyset$.

ii. We have

$$
Q=\bigcup_{k \geq k_{0}(Q)} \bigcup_{P \in \mathcal{F}_{Q}^{k}} P .
$$

iii. We have

$$
\sum_{P \in \mathcal{F}_{Q}^{k}}|P|^{1-s p} \leq C_{16} \lambda_{1}^{\left(k-k_{0}(Q)\right)(\hat{\eta}-D s p)}|Q|^{1-s p} .
$$

B. We have $1_{Q} \in \mathcal{B}_{p, q}^{s}(\mathcal{P})$ for every $q \in[1, \infty]$ and

$$
\left|1_{Q}\right|_{\mathcal{B}_{p, q}^{s}(\mathcal{P})} \leq C_{12}\left(\frac{C_{15}}{C_{14}^{\hat{\eta}} C_{13}^{D}}\right)^{1 / p}|Q|^{1 / p-s}
$$

Proof. Note that

$$
\frac{1}{C_{2}^{2 / D}} \leq \frac{\operatorname{diam}_{d} Q}{\lambda_{1}^{k_{0}(Q)}} \leq \frac{C_{1} C_{6}}{C_{13} \lambda_{1}} .
$$

Let $\mathcal{F}_{Q}^{k} \subset \mathcal{P}^{k}$ be the family of all $P \in \mathcal{P}^{k}$ such that $P \subset Q$ and there is not $P^{\prime} \in \mathcal{P}$ satisfying $P \varsubsetneqq P^{\prime} \subset Q$. For every $P \in \mathcal{F}_{Q}^{k}$ define

$$
s_{P, Q}=|P|^{1 / p-s} \text {. }
$$

otherwise set $s_{P, Q}=0$. Note that

$$
1_{Q}=\sum_{P \in \mathcal{P}} s_{P, Q} b_{P}
$$


Here $b_{Q}$ is the canonical $(s, p)$-Souza's atom on $Q$. If $P \in \mathcal{P}^{k}$ then

$$
m(P) \geq \frac{1}{C_{2}} C_{5}^{D} \lambda_{1}^{D k}
$$

Note also that if $x \in Q$ and

$$
d(x, I \backslash Q)>C_{6} \lambda_{1}^{k-1}
$$

then there is $P \in \mathcal{P}^{k-1}$ such that $x \in P \subset Q$. Consequently if $k \geq k_{0}(Q)$

$$
\begin{aligned}
\sum_{P \in \mathcal{F}_{Q}^{k}}|P| & =m\left(\bigcup_{P \in \mathcal{F}_{Q}^{k}} P\right) \\
& \leq m\left\{x \in Q: d(x, I \backslash Q) \leq C_{6} \lambda_{1}^{k-1}\right\} \\
& \leq C_{15}\left(\frac{C_{6}}{C_{14}} \frac{\lambda_{1}^{k-1}}{\operatorname{diam}_{d} Q}\right)^{\hat{\eta}}|Q| \\
& \leq C_{15}\left(\frac{C_{6} C_{2}^{2 / D} \lambda_{1}^{-1}}{C_{14}}\right)^{\hat{\eta}} \lambda_{1}^{\left(k-k_{0}(Q)\right) \hat{\eta}}|Q| \\
& \leq C_{15} C_{2}\left(\frac{C_{6} C_{2}^{2 / D} \lambda_{1}^{-1}}{C_{14}}\right)^{\hat{\eta}}\left(\frac{C_{1} C_{6}}{C_{13} \lambda_{1}}\right)^{D} \lambda_{1}^{\left(k-k_{0}(Q)\right) \hat{\eta}} \lambda_{1}^{D k_{0}(Q)} \\
& \leq C_{17} \frac{C_{15}}{C_{14}^{\hat{\eta}} C_{13}^{D} \lambda_{1}^{\left(k-k_{0}(Q)\right) \hat{\eta}} \lambda_{1}^{D k_{0}(Q)}} .
\end{aligned}
$$

for some $C_{17} \geq 0$. It follows from (2.11) and (2.14) that

$$
\sharp \mathcal{F}_{Q}^{k} \leq C_{17} \frac{C_{2}}{C_{5}^{D}} \frac{C_{15}}{C_{14}^{\hat{\eta}} C_{13}^{D}} \lambda_{1}^{\left(k-k_{0}(Q)\right)(\hat{\eta}-D)}
$$

Consequently by $(2.11)$

$$
\begin{aligned}
\sum_{P \in \mathcal{F}_{Q}^{k}}\left(\frac{|P|}{|Q|}\right)^{1-s p} & \leq C_{11} \frac{C_{15}}{C_{14}^{\eta} C_{13}^{D}} \lambda_{1}^{\left(k-k_{0}(Q)\right)(\hat{\eta}-D)} \lambda_{1}^{\left(k-k_{0}(Q)\right) D(1-s p)} \\
& \leq C_{11} \frac{C_{15}}{C_{14}^{\eta} C_{13}^{D}} \lambda_{1}^{\left(k-k_{0}(Q)\right)(\hat{\eta}-D s p)}
\end{aligned}
$$

This proves $A$. Together with (2.13) we have that $A$. implies $B$.

Proposition 2.3. Let $\mathcal{P}_{i}, i=\star$, ○, be two grids as in Proposition 2.1 taking $\alpha=d$. Suppose that

$$
\min \left\{\eta_{\star}, \eta_{\circ}\right\}>D s p
$$

Then $\mathcal{B}_{p, q}^{s}\left(\mathcal{P}_{\star}\right)=\mathcal{B}_{p, q}^{s}\left(\mathcal{P}_{\circ}\right)$ and the corresponding norms are equivalent.

Proof. For each $\mathcal{P}_{i}, i=\star$, o denote by $\eta_{i}, C_{5}^{i}, C_{6}^{i}, C_{7}^{i}, C_{8}^{i} \geq 0$ and $\lambda_{1}^{i} \in(0,1)$ the corresponding constants in Proposition 2.1. Fix $Q \in \mathcal{P}_{\star}$. Given $P \in \mathcal{P}_{\circ}$, let $b_{P, Q}=a_{P}$, where $P$ is the canonical Souza's atom supported on $P$. Let $Q \in \mathcal{P}_{\star}^{i}$.

Claim I. We have

$$
k_{i} \leq k_{0}^{\circ}(Q) \leq k_{i}+b
$$

where

$$
k_{i}=i \frac{\ln \lambda_{1}^{\star}}{\ln \lambda_{1}^{\circ}}+\frac{\ln C_{2}^{2}+D\left(\ln C_{6}^{\star}-\ln C_{6}^{\circ}\right)}{D \ln \lambda_{1}^{\circ}} .
$$


Indeed we have

$$
m(Q) \leq C_{2}\left(C_{6}^{\star}\left(\lambda_{1}^{\star}\right)^{i}\right)^{D}
$$

and if $P \in \mathcal{P}_{\circ}^{k}$ then

$$
m(P) \geq C_{2}^{-1}\left(C_{6}^{\circ}\left(\lambda_{1}^{\circ}\right)^{k}\right)^{D} .
$$

In particular if $k<k_{i}$ and $P \in \mathcal{P}_{\circ}^{k}$ then $P \not \subset Q$, so $P \notin \mathcal{F}_{Q}^{k}$ and $s_{P, Q}=0$. In particular $k_{0}^{\circ}(Q) \geq k_{i}$. On the other hand by $(2.8)$

$$
B_{d}\left(z_{Q}^{\star}, C_{18} C_{5}^{\star}\left(\lambda_{1}^{\circ}\right)^{k_{i}}\right) \subset B_{d}\left(z_{Q}^{\star}, C_{5}^{\star}\left(\lambda_{1}^{\star}\right)^{i}\right) \subset Q,
$$

where

$$
C_{18}=\exp \left(-\frac{\ln C_{2}^{2}+D\left(\ln C_{6}^{\star}-\ln C_{6}^{\circ}\right)}{D} .\right.
$$

Let $b \in \mathbb{N}$ be such that

$$
C_{6}^{\circ}\left(\lambda_{1}^{\circ}\right)^{b} \leq C_{18}
$$

Then by (2.9) there is $P$ satisfying $z_{Q}^{\star} \in P \in \mathcal{P}_{\circ}^{k_{i}+b}$ and $P \subset Q$, so (2.16) holds.

Claim II. There is $C_{13}$ such that

$$
B_{d}\left(z_{Q}, C_{13} \operatorname{diam}_{d} Q\right) \subset Q .
$$

By this follows from (2.8) and (2.9) taking $C_{13}=C_{5}^{\star} / C_{6}^{\star}$.

Claim III. There are $C_{14}, C_{15}$ such that

$$
m\left\{x \in Q: d(x, I \backslash Q) \leq C_{14} t \operatorname{diam}_{d} Q\right\} \leq C_{15} t^{\eta_{\star}} m(Q)
$$

holds.

Indeed, by Proposition 2.1 we have

$$
m\left\{x \in Q: d(x, I \backslash Q) \leq C_{7}^{\star} t\left(\lambda_{1}^{\star}\right)^{i}\right\} \leq C_{8}^{\star} t^{\eta_{\star}} m(Q)
$$

By (2.9) we have $\operatorname{diam}_{d} Q \leq C_{6}^{\star}\left(\lambda_{1}^{\star}\right)^{i}$, so

$$
\begin{aligned}
& m\left\{x \in Q: \quad d(x, I \backslash Q) \leq \frac{C_{7}^{\star}}{C_{6}^{\star}} t \operatorname{diam}_{d} Q\right\} \\
\leq & m\left\{x \in Q: \quad d(x, I \backslash Q) \leq C_{7}^{\star} t\left(\lambda_{1}^{\star}\right)^{i}\right\} \leq C_{8}^{\star} t^{\eta_{\star}} m(Q)
\end{aligned}
$$

Claim II and III imply that we can apply Proposition 2.2 for $Q$ and consequently $Q$ is a $\left(1-s p, C_{16},\left(\lambda_{1}^{\circ}\right)^{\eta_{*}-D s p}\right)$-regular domain with respect the good grid $\mathcal{P}^{\circ}$, that is, one can find families $\mathcal{F}_{Q}^{k} \subset \mathcal{P}_{\circ}^{k}$ as in Proposition 2.2 taking $\mathcal{P}=\mathcal{P}_{\circ}$. For every $P \in \mathcal{F}_{Q}^{k}$ define

$$
s_{P, Q}=\left(\frac{|P|}{|Q|}\right)^{1 / p-s} .
$$

otherwise set $s_{P, Q}=0$. Note that

$$
a_{Q}=\sum_{P \in \mathcal{P}_{\circ}} s_{P, Q} a_{P}
$$


and by Claim I

$$
\begin{aligned}
\sum_{P \in \mathcal{F}_{Q}^{k}}\left|s_{P, Q}\right|^{p} & =\sum_{P \in \mathcal{F}_{Q}^{k}}\left(\frac{|Q|}{|P|}\right)^{s p-1} \\
& \leq C_{16}\left(\lambda_{1}^{\circ}\right)^{\left(k-k_{0}^{\circ}(Q)\right)\left(\eta_{\star}-D s p\right)} \\
& \leq C_{19}\left(\lambda_{1}^{\circ}\right)^{\left(k-k_{i}\right)\left(\eta_{\star}-D s p\right)}
\end{aligned}
$$

with $C_{19}=C_{16}\left(\lambda_{1}^{\circ}\right)^{-b\left(\eta_{\star}-D s p\right)}$.

By Proposition 8.1.C in [27] we have that $\mathcal{B}_{p, q}^{s}\left(\mathcal{P}_{\star}\right) \subset \mathcal{B}_{p, q}^{s}\left(\mathcal{P}_{\circ}\right)$ and there exists $C$ such that

$$
|f|_{\mathcal{B}_{p, q}^{s}\left(\mathcal{P}_{\circ}\right)} \leq C|f|_{\mathcal{B}_{p, q}^{s}\left(\mathcal{P}_{\star}\right)}
$$

for every $f \in \mathcal{B}_{p, q}^{s}\left(\mathcal{P}_{\star}\right)$. Exchanging the roles of $\mathcal{P}_{\star}$ and $\mathcal{P}_{\circ}$ in the above argument we obtain the reverse inclusion and inequality.

Definition 2.4. Let $(I, \rho, m)$ be a homogeneous space and $(m, d, 1)$ be a Ahlfors regular quasi-metric space such that $d$ is power-law equivalent to $\rho$. Let $\tilde{\eta}$ be the supremum of all $\eta$ that admits a good grid as in Proposition 2.1 with $\alpha=d$. Let $s \in(0,1 / p), p \in[1, \infty)$ and $q \in[1, \infty]$. Assume $\tilde{\eta}>s p$. We define the Besov space $\mathbb{B}_{p, q}^{s}$ on the homogeneous space $(I, \rho, m)$ as $\mathcal{B}_{p, q}^{s}(\mathcal{P})$. The Besov space $\mathbb{B}_{p, q}^{s}$ is well-defined due Proposition 2.3.

Han, Lu and Yang [15] defined inhomogeneous Besov spaces $B_{H L Y}(s, p, q)$ for homogeneous spaces introducing a type of Calderón reproducing formula. They also obtained an atomic decomposition of these Besov spaces, that we describe now. Let $\beta$ as in (2.5) and $C_{20}$ be such that

$$
\beta(x, z) \leq C_{20}(\beta(x, y)+\beta(y, z))
$$

Note that $(I, m, \beta, 1)$ is an Ahlfors regular quasi-metric space. Let $\mathcal{P}^{i}$ be a grid as in Proposition 2.1 taking $\alpha=\beta$. Fix $\gamma>0$ small enough such that $d=d_{\gamma}$ as defined in (2.7) is a metric satisfying

$$
\frac{1}{C_{21}} d_{\gamma}(x, y) \leq \beta^{\gamma}(x, y) \leq C_{21} d_{\gamma}(x, y)
$$

Let $s>0$ be such that $0<s<\gamma$. A Han-Lu-Yang $\gamma$-block $a_{Q}$ associated to $Q \in \mathcal{P}^{k}$ is a function

$$
a_{Q}: I \rightarrow \mathbb{C}
$$

such that

i. supp $a_{Q} \subset B_{\beta}\left(z_{Q}, 3 C_{20} C_{6} \lambda_{1}^{k}\right)$.

ii. $\left|a_{Q}\right|_{\infty} \leq|Q|^{s-1 / p}$.

iii. $\left|a_{Q}(x)-a_{Q}(y)\right| \leq|Q|^{s-1 / p-\gamma} \beta(x, y)^{\gamma}$.

A function $f \in L^{p}$ belongs to $B_{H L Y}(s, p, q)$ if (Theorem 2.1 and Proposition 3.1 in [15], and also Theorem 6.5 and Remark 6.20 in Han and Sawyer [16]) we can write

$$
f=\sum_{k} \sum_{Q \in \mathcal{P}^{k}} c_{Q} a_{Q}
$$


D. SMANIA

where $a_{Q}$ is a Han-Lu-Yang $\gamma$-block $a_{Q}$ associated to $Q$ and the convergence is absolute in $L^{p}$, that is

$$
\sum_{k}\left|\sum_{Q \in \mathcal{P}^{k}} c_{Q} a_{Q}\right|_{p}<\infty
$$

and

$$
\left(\sum_{k}\left(\sum_{Q \in \mathcal{P}^{k}}\left|c_{Q}\right|^{p}\right)^{q / p}\right)^{1 / q}<\infty
$$

We define

$$
|f|_{B_{H L Y}(s, p, q)}=\inf \left(\sum_{k}\left(\sum_{Q \in \mathcal{P}^{k}}\left|c_{Q}\right|^{p}\right)^{q / p}\right)^{1 / q}
$$

where the infimum runs over all possible representations (2.23). We are using a slightly different definition for an $\gamma$-block but we also modified the norm definition accordingly to obtain the same Besov space as in Han, Lu and Yang [15].

Proposition 2.5. Let $\mathcal{P}$ be a good grid as in Proposition 2.1 with $\alpha=\beta$. Suppose that $\eta>s p, 0<s<\gamma, p \in[1, \infty)$ and $q \in[0, \infty]$. Then $B_{H L Y}(s, p, q)=\mathbb{B}_{p, q}^{s}(\mathcal{P})$.

Proof. We will prove it in several steps.

Step I. Consider the grid

$$
\mathcal{F}^{k}=\left\{B_{\beta}\left(z_{Q}, 3 C_{20} C_{6} \lambda_{1}^{k}\right): Q \in \mathcal{P}^{k}\right\} .
$$

Let $\mathcal{A}_{s, p}^{H L Y}\left(B_{\beta}\left(z_{Q}, 3 C_{20} C_{6} \lambda_{1}^{k}\right)\right)$ be the class of atoms that consists of functions $\phi$ that satisfy

i. supp $\phi \subset B_{\beta}\left(z_{Q}, 3 C_{20} C_{6} \lambda_{1}^{k}\right)$.

ii. $|\phi|_{\infty} \leq\left|B_{\beta}\left(z_{Q}, 3 C_{20} C_{6} \lambda_{1}^{k}\right)\right|^{s-1 / p}$.

iii. $|\phi(x)-\phi(y)| \leq|Q|^{s-1 / p-\gamma} \beta(x, y)^{\gamma}$.

Note that there is $C_{22}>1$ such that for every $Q \in \mathcal{P}$

$$
\frac{1}{C_{22}} \leq \frac{\left|B_{\beta}\left(z_{Q}, 3 C_{20} C_{6} \lambda_{1}^{k}\right)\right|}{|Q|} \leq C_{22}
$$

This implies that $B_{H L Y}(s, p, q)=\mathcal{B}_{p, q}^{s}\left(\mathcal{A}_{s, p}^{H L Y}\right)$ and its norms are equivalent. We will prove that $\mathcal{B}_{p, q}^{s}\left(\mathcal{A}_{s, p}^{H L Y}\right)=\mathcal{B}_{p, q}^{s}$.

Step II. Let $e_{Q}$ be the canonical $(s, p)$-Souza's atom on $Q$. We claim that $e_{Q} \in$ $\mathcal{B}_{p, q}^{s}\left(\mathcal{A}_{s, p}^{H L Y}\right)$ for every $Q \in \mathcal{P}^{k_{0}}, k_{0} \geq 1$. Indeed for every $k \geq k_{0}$, let $\mathcal{F}^{k}(Q)$ be the family of all $P \in \mathcal{P}^{k}$ such that

A. We have

$$
P \subset B_{\beta}\left(z_{P}, 3 C_{20} C_{6} \lambda_{1}^{k}\right) \subset Q .
$$

B. If $P \subset W \in \mathcal{P}^{i}$, with $i<k$ we have

$$
B_{\beta}\left(z_{W}, 3 C_{20} C_{6} \lambda_{1}^{i}\right) \not \subset Q .
$$

There is $C_{23}>0$ such that for every $x \in B_{\beta}\left(z_{P}, 2 C_{6} \lambda_{1}^{k}\right)$, with $P \in \mathcal{F}^{k}(Q)$, we have

$$
\frac{1}{C_{23}} \lambda_{1}^{k} \leq d_{\beta}(x, I \backslash Q) \leq C_{23} \lambda_{1}^{k} .
$$


In particular by (2.10) there is $C_{24}, C_{25}$ such that

$$
m\left(\bigcup_{P \in \mathcal{F}^{k}(Q)} P\right) \leq C_{24} \lambda_{1}^{\eta\left(k-k_{0}\right)}|Q| \leq C_{25} \lambda_{1}^{k_{0}+\eta\left(k-k_{0}\right)}
$$

for every $k$, and consequently

$$
\# \mathcal{F}^{k}(Q) \leq C_{26} \lambda_{1}^{(\eta-1)\left(k-k_{0}\right)}
$$

for some constant $C_{26}$. Note that

$$
Q=\bigcup_{k \geq k_{0}} \bigcup_{P \in \mathcal{F}^{k}(Q)} P=\bigcup_{k \geq k_{0}} \bigcup_{P \in \mathcal{F}^{k}(Q)} B_{\beta}\left(z_{P}, 2 C_{6} \lambda_{1}^{k}\right) .
$$

For every $P \in \mathcal{F}^{k}(Q)$ such that $I \backslash B_{\beta}\left(z_{P}, 2 C_{6} \lambda_{1}^{k}\right) \neq \emptyset$ define

$$
\psi_{P}(x)=|P|^{-\gamma} d_{\gamma}\left(x, I \backslash B_{\beta}\left(z_{P}, 2 C_{6} \lambda_{1}^{k}\right)\right),
$$

otherwise let $\psi_{P}(x)=1$. There is $C_{27}>1$ such that for every $P \in \mathcal{F}^{k}(Q), k \geq 1$, we have

$$
\psi_{P}(x) \leq C_{27}
$$

for every $x \in I$ and

$$
\frac{1}{C_{27}} \leq \psi_{P}(x)
$$

for every $x \in P$. We claim that

$$
\left|\psi_{P}(x)-\psi_{P}(y)\right| \leq 2 C_{27} C_{21}|P|^{-\gamma} \beta(x, y)^{\gamma}
$$

for every $x, y \in I$. Indeed, if $d_{\gamma}(x, y) \geq|P|^{\gamma}$ then

$$
\left|\psi_{P}(x)-\psi_{P}(y)\right| \leq 2 C_{27} \leq 2 C_{27}|P|^{-\gamma} d_{\gamma}(x, y) \leq 2 C_{27} C_{21}|P|^{-\gamma} \beta(x, y)^{\gamma},
$$

and if $d_{\gamma}(x, y) \leq|P|^{\gamma}$ then

$$
\left|\psi_{P}(x)-\psi_{P}(y)\right| \leq|P|^{-\gamma} d_{\gamma}(x, y) \leq C_{21}|P|^{-\gamma} \beta(x, y)^{\gamma} .
$$

In particular

is a $\gamma$-block. Let

$$
a_{P}(x)=\frac{1}{2 C_{27} C_{21}}|P|^{s-1 / p} \psi_{P}(x)
$$

$$
f_{Q}(x)=\sum_{k \geq k_{0}} \sum_{P \in \mathcal{F}^{k}(Q)} 2 C_{27} C_{21}|P|^{1 / p-s} a_{P}
$$

Note that $f_{Q}(x)=0$ if $x \notin Q$ and $f_{Q} \in \mathcal{B}_{p, q}^{s}\left(\mathcal{A}_{s, p}^{H L Y}\right)$, since by (2.24) there is $C_{28}, C_{29}$ such that

$$
\begin{aligned}
\sum_{P \in \mathcal{F}^{k}(Q)}|P|^{1-s p} & \leq \sum_{P \in \mathcal{F}^{k}(Q)}\left(C_{6} \lambda_{1}^{k}\right)^{1-s p} \\
& \leq C_{6}^{1-s p} C_{26} \lambda_{1}^{(\eta-1)\left(k-k_{0}\right)} \lambda_{1}^{k(1-s p)} \\
& \leq C_{6}^{1-s p} C_{26} \lambda_{1}^{(\eta-s p)\left(k-k_{0}\right)} \lambda_{1}^{(1-s p) k_{0}} \\
& \leq C_{29} C_{6}^{1-s p} C_{26} \lambda_{1}^{(\eta-s p)\left(k-k_{0}\right)}|Q|^{1-s p}
\end{aligned}
$$


D. SMANIA

and consequently

$$
\begin{aligned}
\left(\sum_{k \geq k_{0}}\left(\sum_{P \in \mathcal{F}^{k}(Q)}|P|^{1-s p}\right)^{q / p}\right)^{1 / q} & \leq C_{29}^{1 / p} C_{6}^{1 / p-s} C_{26}^{1 / p}\left(\sum_{k \geq k_{0}} \lambda_{1}^{\frac{q}{p}(\eta-s p)\left(k-k_{0}\right)}\right)^{1 / q}|Q|^{1 / p-s} . \\
& \leq C_{28}|Q|^{1 / p-s} .
\end{aligned}
$$

Finally note that due (2.6) and (2.8) there is $C_{30}, C_{31}$ such that for every $P \in$ $\mathcal{F}^{k}(Q), k \geq 1$, we have

$$
\# \cup_{j}\left\{R \in \mathcal{F}^{j}(Q) \text { s.t. } B_{\beta}\left(z_{R}, 2 C_{6} \lambda_{1}^{j}\right) \cap B_{\beta}\left(z_{P}, 2 C_{6} \lambda_{1}^{k}\right) \neq \emptyset\right\} \leq C_{30}
$$

and $|j-k| \leq C_{31}$ for every $j$ such that there is $R \in \mathcal{F}^{j}(Q)$ satisfying

$$
B_{\beta}\left(z_{R}, 2 C_{6} \lambda_{1}^{j}\right) \cap B_{\beta}\left(z_{P}, 2 C_{6} \lambda_{1}^{k}\right) \neq \emptyset
$$

for some $P \in \mathcal{F}^{k}(Q)$. As a consequence $f_{Q}$ satisfies

$$
\left|f_{Q}(x)-f_{Q}(y)\right| \leq C_{32}|P|^{-\gamma} \beta(x, y)^{\gamma}
$$

for every $x, y \in B_{\beta}\left(z_{P}, 2 C_{6} \lambda_{1}^{k}\right)$, with $P \in \mathcal{F}^{k}, k \geq 1$. Moreover (2.26) and (2.27) implies

for every $x \in Q$. This implies that

$$
\frac{1}{C_{27}} \leq f_{Q}(x) \leq C_{27} C_{30}
$$

$$
\left|\frac{1}{f_{Q}(x)}-\frac{1}{f_{Q}(y)}\right| \leq C_{33}|P|^{-\gamma} \beta(x, y)^{\gamma}
$$

for every $x, y \in B_{\beta}\left(z_{P}, 2 C_{6} \lambda_{1}^{k}\right)$, with $P \in \mathcal{F}^{k}, k \geq 1$. Then

$$
b_{P, Q}(x)=\frac{\psi_{P}(x)}{\left(C_{27} C_{33}+2 C_{27}^{2} C_{21}\right) f_{Q}(x)} .
$$

is a $\gamma$-block on $Q$ and

$$
1_{Q}=\sum_{k} \sum_{P \in \mathcal{F}^{k}(Q)} C_{34}|P|^{1 / p-s} b_{P, Q} .
$$

where $C_{34}=C_{27} C_{33}+2 C_{27}^{2} C_{21}$. So (2.29) implies that $1_{Q} \in \mathcal{B}_{p, q}^{s}\left(\mathcal{A}_{s, p}^{H L Y}\right)$. So if $e_{Q}$ is the canonical $(s, p)$-Souza's atom on $Q$ then

$$
e_{Q}=\sum_{k} \sum_{P \in \mathcal{F}^{k}(Q)} r_{P, Q} b_{P, Q}
$$

where

$$
r_{P, Q}=C_{34}\left(\frac{|P|}{|Q|}\right)^{1 / p-s}
$$

Due (2.28)

$$
\sum_{P \in \mathcal{F}^{k}(Q)} r_{P, Q}^{p} \leq C_{35} \lambda_{1}^{(\eta-s p)\left(k-k_{0}\right)}
$$

In particular $e_{Q} \in \mathcal{B}_{p, q}^{s}\left(\mathcal{A}_{s, p}^{H L Y}\right)$.

Step III. We apply Proposition 8.1.C in [27] with $\mathcal{A}_{2}=\mathcal{A}_{s, p}^{H L Y}, \mathcal{A}_{1}=\mathcal{A}_{s, p}^{s z}, \mathcal{G}=\mathcal{P}$ and $\mathcal{W}=\mathcal{F}$, and $k_{i}=i$ for every $i$. We have that assumption (8.14) in [27] follows from (2.30). Consequently

$$
\mathcal{B}_{p, q}^{s}\left(\mathcal{A}_{s, p}^{s z}\right) \subset \mathcal{B}_{p, q}^{s}\left(\mathcal{A}_{s, p}^{H L Y}\right)
$$


and this inclusion is continuous.

Step IV. To prove the reverse continuous inclusion, note that there is $C_{36}$ such that

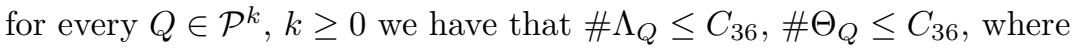

$$
\Lambda_{Q}=\left\{P \in \mathcal{P}^{k}: P \cap B_{\beta}\left(z_{Q}, 3 C_{20} C_{6} \lambda_{1}^{k}\right) \neq \emptyset\right\},
$$

and

$$
\Theta_{Q}=\left\{P \in \mathcal{P}^{k}: Q \cap B_{\beta}\left(z_{P}, 3 C_{20} C_{6} \lambda_{1}^{k}\right) \neq \emptyset\right\} .
$$

There is $C_{37}>0$ such that the following holds. If $\phi_{Q} \in \mathcal{A}_{s, p}^{H L Y}\left(B_{\beta}\left(z_{Q}, 3 C_{20} C_{6} \lambda_{1}^{k}\right)\right)$ then for every $P \in \Lambda_{Q}$ we have

$$
C_{37} \phi_{Q} 1_{P} \in \mathcal{A}_{s, 1, p}^{h}(P)
$$

where $\mathcal{A}_{s, 1, p}^{h}$ are as defined in Section 11.2 in [27] taking $d=\beta, D=1$ and $\beta=\gamma$. So if

$$
g=\sum_{k} \sum_{Q \in \mathcal{P}^{k}} c_{Q} \phi_{Q}
$$

is a $\mathcal{B}_{p, q}^{s}\left(\mathcal{A}_{s, p}^{H L Y}\right)$-representation then define

$$
m_{P}=\frac{1}{C_{37}} \sum_{P \in \Lambda_{Q}}\left|c_{Q}\right|=\frac{1}{C_{37}} \sum_{Q \in \Theta_{P}}\left|c_{Q}\right| .
$$

We have that

$$
\psi_{P}=\frac{1}{m_{P}} \sum_{P \in \Lambda_{Q}} C_{37} c_{Q} \phi_{Q} 1_{P} \in \mathcal{A}_{s, 1, p}^{h}(P)
$$

and

$$
g=\sum_{k} \sum_{P \in \mathcal{P}^{k}} m_{P} \psi_{P}
$$

is a $\mathcal{B}_{p, q}^{s}\left(\mathcal{A}_{s, 1, p}^{h}\right)$-representation of $g$. Indeed

$$
\begin{aligned}
\sum_{P \in \mathcal{P}^{k}}\left|m_{P}\right|^{p} & =\frac{1}{C_{37}^{p}} \sum_{P \in \mathcal{P}^{k}}\left(\sum_{Q \in \Theta_{P}}\left|c_{Q}\right|\right)^{p} \\
& \leq \frac{C_{36}^{p}}{C_{37}^{p}} \sum_{P \in \mathcal{P}^{k}} \sum_{Q \in \Theta_{P}}\left|c_{Q}\right|^{p} \\
& \leq \frac{C_{36}^{p}}{C_{37}^{p}} \sum_{Q \in \mathcal{P}^{k}} \sum_{P \in \Lambda_{Q}}\left|c_{Q}\right|^{p} \\
& \leq \frac{C_{36}^{p+1}}{C_{37}^{p}} \sum_{Q \in \mathcal{P}^{k}}\left|c_{Q}\right|^{p} .
\end{aligned}
$$

So $g \in \mathcal{B}_{p, q}^{s}\left(\mathcal{A}_{s, 1, p}^{h}\right)$ and

$$
|g|_{\mathcal{B}_{p, q}^{s}\left(\mathcal{A}_{s, 1, p}^{h}\right)} \leq \frac{C_{36}^{1+1 / p}}{C_{37}}|g|_{\mathcal{B}_{p, q}^{s}\left(\mathcal{A}_{s, p}^{H L Y}\right)}
$$

Since by Proposition 16.2 in [27] we have that $\mathcal{B}_{p, q}^{s}\left(\mathcal{A}_{s, 1, p}^{h}\right)=\mathcal{B}_{p, q}^{s}$ and their norms are equivalent. The proof is complete. 
2.4. The case $[0,1]^{D}$. We would like to connect this abstract setting with classical Besov spaces on $\mathbb{R}^{D}$ considering the very well-known homogeneous space $\left([0,1]^{D}, m, d\right)$, where $d$ is the euclidean metric on $\mathbb{R}^{D}$ and $m$ is the Lebesgue measure on $\mathbb{R}^{D}$. Then $\left(m, d^{D}, 1\right)$ is a Ahlfors regular quasi-metric space. Note that we can choose $\gamma=1 / D$. Let $\mathcal{P}^{k}$ be the partition of $[0,1]^{D}$ by $D$-dimensional cubes with 1-dimensional faces with length $2^{-k}$ which are parallels to the coordinate axes. Then $\left(\mathcal{P}^{k}\right)_{k}$ is a good grid satisfying the conclusion of Proposition 2.1 when $\alpha=d^{D}$ and taking $\lambda_{1}=1 / 2^{D}$ and $\eta=1 / D$. By Proposition 2.3 we have that $\mathbb{B}_{p, q}^{s}$ is welldefined provided $p \in[1, \infty), q \in[1, \infty]$ and $0<s<1 /(D p)$. Proposition 2.5 implies that $B_{H L Y}(s, p, q)=\mathbb{B}_{p, q}^{s}$ for every $p \in[1, \infty), q \in[1, \infty]$ and $0<s<1 /(D p)$.

Consider the classical inhomogeneous Besov space $B_{\text {class }}(\hat{s}, p, q)$ of $\mathbb{R}^{D}$, with $0<\hat{s}<1 / p, p \in[1, \infty)$ and $q \in[1, \infty]$. Let

$$
T=\left\{f 1_{[0,1]^{D}}: f \in B_{\text {class }}(s D, p, q)\right\} .
$$

We believe that it is well know that $B_{H L Y}(s, p, q)=T$, however since we did not find a reference we provide a proof.

Proposition 2.6. We have $T=\mathbb{B}_{p, q}^{s}$, for every $s<1 /(D p), p \in[1, \infty)$ and $q \in[1, \infty]$.

Proof. Fix $M>1$. It follows from Frazier and Jawerth [13] that $\mathrm{T}$ is the set of all functions $f$ that can be written as in (1.1), where $s_{Q}$ satisfy (1.2) and $a_{Q}$ are $C^{1}$ functions in $\mathbb{R}^{D}$ such that supp $a_{Q} \subset M Q$,

$$
\left|a_{Q}\right| \leq|Q|^{\hat{s} / D-1 / p}
$$

and

$$
\left|\partial_{i} a_{Q}\right| \leq|Q|^{\hat{s} / D-1 / p-1 / D}
$$

for every $i=1, \ldots, D$. In particular

$$
\left|a_{Q}(x)-a_{Q}(y)\right| \leq C_{38}|Q|^{\hat{s} / D-1 / p-1 / D}|x-y|
$$

for some constant $C_{38}$. Due the atomic decomposition of $B_{H L Y}(s, p, q)$ by $\mathrm{Han}, \mathrm{Lu}$ and Yang [15] we conclude that $T \subset B_{H L Y}(s, p, q)=\mathbb{B}_{p, q}^{s}$ (recall that $\left.|x-y|=\beta(x, y)^{\gamma}\right)$.

To show that $\mathbb{B}_{p, q}^{s} \subset T$ we need to adapt the proof of Proposition 2.5, firstly considering all functions and balls defined in the whole $\mathbb{R}^{D}$ (opposite to just on $\left.[0,1]^{D}\right)$. If we modify the definition of $\mathcal{A}_{s, p}^{H L Y}\left(B_{\beta}\left(z_{Q}, 3 C_{20} C_{6} \lambda_{1}^{k}\right)\right)$ in Step I requiring additionally that all functions in there must be $C^{1}$ on $\mathbb{R}^{D}$ then $T=\mathcal{B}_{p, q}^{s}\left(\mathcal{A}_{s, p}^{H L Y}\right)$. Moreover on Step II. we need to modify the definition of $\psi_{P}$ to get a $C^{1}$ function on $\mathbb{R}^{D}$. Replace $(2.25)$ by

$$
\psi_{P}(x)=\frac{1}{2}|P|^{-2 \gamma} d_{\gamma}^{2}\left(x, I \backslash B_{\beta}\left(z_{P}, 2 C_{6} \lambda_{1}^{k}\right)\right),
$$

Since $d_{\gamma}$ is just the euclidean distance and $B_{\beta}\left(z_{P}, r\right)$, with $r>0$, is an euclidean ball, we have that $\psi_{P}$ is $C^{1}$ on $\mathbb{R}^{D}$ and it satisfies all the estimates in the proof. Then we can carry out Step III to conclude that $\mathbb{B}_{p, q}^{s} \subset T$.

\section{Gu-Taibleson recalibrated martingale Besov spaces}

Let $I$ be a compact Hausdorff space and let $m$ be a borelian measure such that $m(I)=1$. If $\mathcal{P}$ is a good grid on $I$, we can consider the recalibrated martingale Besov spaces as defined by $\mathrm{Gu}$ and Taibleson [14]. In what follows, we just made 
the obvious adaptations of their definition to the compact setting. Instead of dealing directly with the good grid $\mathcal{P}$ to define $\mathcal{B}_{p, q}^{s}(\mathcal{P}), \mathrm{Gu}$ and Taibleson define a recalibration of the martingale structure as follows. A recalibration of $\mathcal{P}$ is a new good grid $\mathcal{G}$ such that

$$
\cup_{k} \mathcal{G}^{k} \subset \cup_{k} \mathcal{F}^{k}
$$

and satisfying the following properties. If

$$
\ell_{k}=\max _{P \in \mathcal{G}^{k}}|P|, m_{k}=\min _{P \in \mathcal{G}^{k}}|P|
$$

then

$$
\ell_{k+1}<\ell_{k}
$$

and there exist $\lambda_{3}, \lambda_{4}, \delta \in(0,1)$ such that

$$
0<\lambda_{3} \leq \frac{\ell_{k+1}}{\ell_{k}} \leq \lambda_{4}<1
$$

and

$$
1 \leq \frac{\ell_{k}}{m_{k}} \leq \frac{1}{\delta}
$$

for every $k$. By Gu and Taibleson [14] a recalibration always exists.

Given $f \in L_{1}$, define

$$
f_{k}(x)=\frac{1}{|Q|} \int_{Q} f d m
$$

for every $x \in Q \in \mathcal{G}^{k}$ and

$$
d_{k} f(x)=f_{k}(x)-f_{k-1}(x) .
$$

Of course

$$
\int_{Q} d_{k} f d m=0
$$

for every $Q \in \mathcal{G}^{k-1}$. Note that $f_{k}$ is exactly the function $f_{k}$ defined in (17.46) in [27], if we replace $\mathcal{P}^{k}$ by $\mathcal{G}^{k}$ there. We say that that $f$ belongs to the $\mathbf{G u}$-Taibleson recalibrated martingale Besov space $B_{G T}(s, p, q)$ if

$$
|f|_{B_{G T}(s, p, q)}=\left(\sum_{k}\left(\ell_{k}^{-s}\left|d_{k} f\right|_{p}\right)^{q}\right)^{1 / q} \sim\left(\sum_{k}\left(\ell_{k}^{-s}\left|f-f_{k}\right|_{p}\right)^{q}\right)^{1 / q}<\infty .
$$

Gu and Taibleson also proved that

$$
|f|_{B_{G T}(s, p, q)} \sim\left(\sum_{k}\left(\ell_{k}^{-s}\left|f-f_{k}\right|_{p}\right)^{q}\right)^{1 / q} .
$$

Proposition 3.1. We have $B_{G T}(s, p, q)=\mathcal{B}_{p, q}^{s}(\mathcal{G})$ and its norms are equivalent. 
Proof. By Theorem 15.1 in [27] we have

$$
\begin{aligned}
|f|_{\mathcal{B}_{p, q}^{s}(\mathcal{G})} & \sim\left(\sum_{k}\left(\sum_{Q \in \mathcal{P}^{k}}|Q|^{-s p} \operatorname{osc}_{p}(f, Q)^{p}\right)^{q / p}\right)^{1 / q} \\
& \leq\left(\sum_{k}\left(\sum_{Q \in \mathcal{P}^{k}}|Q|^{-s p} \int_{Q}\left|f-f_{k-1}\right|^{p} d m\right)^{q / p}\right)^{1 / q} \\
& \leq\left(\sum_{k}\left(\sum_{Q \in \mathcal{P}^{k}} \ell_{k}^{-s p} \int_{Q}\left|f-f_{k-1}\right|^{p} d m\right)^{q / p}\right)^{1 / q} \\
& \leq\left(\sum_{k}\left(\ell_{k}^{-s}\left|f-f_{k-1}\right|_{p}\right)^{q}\right)^{1 / q} \sim|f|_{B_{G T}(s, p, q)} .
\end{aligned}
$$

On the other hand by (14.25) and Theorem 15.1 in [27]

$$
\begin{aligned}
\left(\sum_{k}\left(\ell_{k}^{-s}\left|d_{k} f\right|_{p} \mid\right)^{q}\right)^{1 / q} & =\left(\sum_{k}\left(\sum_{Q \in \mathcal{G}^{k}} \ell_{k}^{-s p} \int_{Q}\left|d_{k} f\right|^{p} d m\right)^{q / p}\right)^{1 / q} \\
& \leq C\left(\sum_{k}\left(\sum_{Q \in \mathcal{G}^{k}}|Q|^{-s p} \int_{Q}\left|d_{k} f\right|^{p}\right)^{q / p}\right)^{1 / q} \\
& \leq C\left(\sum_{k}\left(\sum_{Q \in \mathcal{G}^{k}}|Q|^{-s p} \int_{Q}\left|\sum_{S \in \mathcal{H}_{Q}} d_{S} \phi_{S}\right|^{p} d m\right)^{q / p}\right)^{1 / q} \\
& \leq \hat{C}\left(\sum_{k}\left(\sum_{Q \in \mathcal{G}^{k}}|Q|^{-s p} \int_{Q} \sum_{S \in \mathcal{H}_{Q}}\left|d_{S}\right|^{p}\left|\phi_{S}\right|^{p} d m\right)^{q / p}\right)^{1 / q} \\
& \leq \tilde{C}\left(\sum_{k}\left(\sum_{Q \in \mathcal{G}^{k}}|Q|^{1-s p-p / 2} \sum_{S \in \mathcal{H}_{Q}}\left|d_{S}\right|^{p}\right)^{q / p}\right)^{1 / q} \\
& \leq C^{\prime}|f|_{\mathcal{B}_{p, q}^{s}} .
\end{aligned}
$$

Following $\mathrm{Gu}$ and Taibleson, we can endow $I$ with the metric

$$
\rho(x, y)=\inf \{|Q|, Q \in \mathcal{P} \text { and } x, y \in Q\} .
$$

Then it is easy to see that $(I, \rho, m)$ is a 1-Ahlfors regular space, so in particular a homogeneous space. Indeed in this case $\rho=\beta=d_{1}$, where $\beta$ and $d_{1}$ are as in (2.5) and (2.7). The following result is, as far as we know, new, and says that a GuTaibleson recalibrated martingale Besov spaces are particular examples of Besov spaces on homogenous spaces.

Theorem 3.2. For $s>0$ small and $p, q \geq 1$ the Gu-Taibleson recalibrated martingale Besov space $B_{G T}(s, p, q)$ does not depend on the chosen recalibration and it coincides with the Besov space $\mathbb{B}_{p, q}^{s}=\mathcal{B}_{p, q}^{s}(\mathcal{G})=B_{H L Y}(s, p, q)$ of the homogeneous space $(I, \rho, m)$.

Proof. If $C>0$ is large enough and $\lambda \in(0,1)$ is small enough, there exists an increasing sequence $k_{i}$ such that

$$
\frac{1}{C} \lambda^{i} \leq|Q| \leq C \lambda^{i}
$$




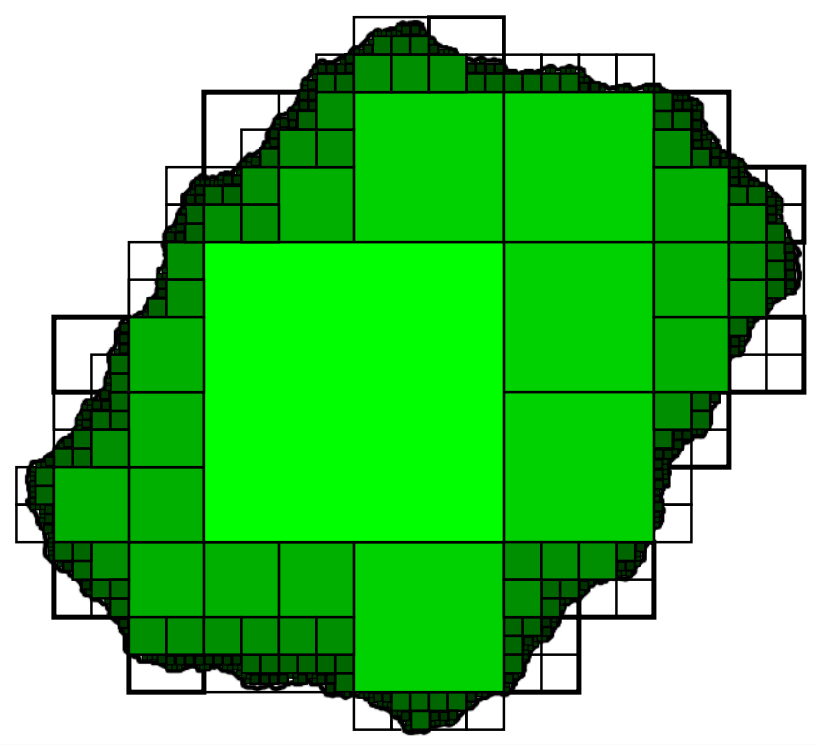

Figure 1. A Julia set $J$ of a quadratic polynomial $x^{2}+c$, with $c$ small, is a quasicircle. Every quasi-circle delimits a regular domain, but indeed $J$ is the boundary of a strongly regular domain.

for every $Q \in \mathcal{G}^{k_{i}}$. Define the new grid (which may not be a good grid) $\hat{\mathcal{W}}$ as $\hat{\mathcal{W}}^{k_{i}}=\mathcal{G}^{k_{i}}$ for every $i$ and $\hat{\mathcal{W}}^{k}=\emptyset$ if $k \notin\left\{k_{i}\right\}_{i}$. One can easily prove using Proposition 8.1 in [27] that $\mathcal{B}_{p, q}^{s}(\hat{\mathcal{W}})=\mathcal{B}_{p, q}^{s}(\mathcal{G})=B_{G T}(s, p, q)$ and theirs norms are equivalent. Define the good grid $\mathcal{W}$ as $\mathcal{W}^{i}=\hat{\mathcal{W}}^{k_{i}}$. We have $\mathcal{B}_{p, q}^{s}(\mathcal{W})=\mathcal{B}_{p, q}^{s}(\hat{\mathcal{W}})$. Note that $\mathcal{W}$ is a good grid as in Proposition 2.1 taking $\lambda_{1}=\lambda$. Indeed (2.8) and (2.9) are easy to verify. Note that if $x \in Q \in \mathcal{W}$ and $y \notin Q$ we have

$$
d(x, y) \geq|Q| \geq \frac{1}{C} \lambda^{i} .
$$

and so (2.10) holds and consequently (see Definition 2.4) we have $\mathcal{B}_{p, q}^{s}(\mathcal{W})=\mathbb{B}_{p, q}^{s}=$ $B_{H L Y}(s, p, q)$.

We are going to see in Remark 7.3 that it is possible to choose the initial good grid $\mathcal{P}$ is such way that $\mathcal{B}_{p, q}^{s}(\mathcal{P})$ is not $\mathbb{B}_{p, q}^{s}(I, \rho, m)$. That means that the families of spaces defined by Souza's atoms is richer than the families of Besov spaces on homogeneous spaces and recalibrated martingale Besov spaces.

\section{EXAMPLES OF STRONGLY REgUlaR DOMAINS}

Let's assume that $(I, d, m, D)$ is an Ahlfors quasi-metric space, such that (2.4) holds for every $x \in I$ and $r \leq r_{0}$. The following result give sufficient geometric conditions on $\partial \Omega$ for $\Omega$ to be a strongly regular domain, and in particular for $1_{\Omega}$ to define a multiplier in appropriated Besov spaces (see Section 4). This is obviously a generalisation of results in Triebel[29] to our setting.

Proposition 4.1. Let $\mathcal{P}$ be good grid for $(I, d, m, D)$ as in Proposition 2.1. Let $K \subset I$ be a closed subset such that $(K, d, \mu, \alpha)$ is Ahlfors regular quasi-metric-space, 
with $\alpha<D$ and some finite borelian measure $\mu$, that is, for every $x \in K$ and $r \leq r_{1}$

$$
\frac{1}{C_{39}} r^{\alpha} \leq \mu\left(B_{d}(x, r)\right) \leq C_{39} r^{\alpha} .
$$

Then $K^{c}$ is a $\left(\alpha / D, C_{40}, C_{41}\right)$-strongly regular domain for some $C_{40} \geq 0$ and $C_{41}$ satisfying

$$
4 C_{6} \lambda_{1}^{C_{41}-1}<\min \left\{r_{0}, r_{1}\right\},
$$

that is, for every $Q \in \mathcal{P}$ such that $k_{0}(Q) \geq C_{41}$ one can find families $\mathcal{F}^{k}\left(Q \cap K^{c}\right) \subset \mathcal{P}^{k}$, $k \geq k_{0}(Q)$, such that

A. We have

$$
Q \cap K^{c}=\cup_{k \geq k_{0}(Q)} \cup_{P \in \mathcal{F}^{k}\left(Q \cap K^{c}\right)} P
$$

B. If $P, J \in \cup_{k \geq k_{0}(Q)} \mathcal{F}^{k}\left(Q \cap K^{c}\right)$ and $P \neq J$ then $P \cap J=\emptyset$.

C. We have

$$
\sum_{P \in \mathcal{F}^{k}\left(Q \cap K^{c}\right)}|P|^{\alpha / D} \leq C_{40}|Q|^{\alpha / D} .
$$

Suppose additionally that every element of $\mathcal{P}$ is a connected set. Then every subset $\Omega \subset K^{c}$ such that $\partial \Omega \subset K$ is also a $\left(\alpha / D, C_{40}\right)$-strongly regular domain.

Proof. Suppose $Q \cap K \neq \emptyset$, otherwise there is nothing to do. Define

$$
\mathcal{F}^{k_{0}(Q)}\left(Q \cap K^{c}\right)=\left\{P \in \mathcal{P}^{k_{0}}, P \subset Q \cap K^{c}\right\} .
$$

and for $k>k_{0}(Q)$

$$
\mathcal{F}^{k}\left(Q \cap K^{c}\right)=\left\{P \in \mathcal{P}^{k}, P \subset\left(Q \cap K^{c}\right) \backslash \cup_{k_{0} \leq i<k} \cup_{P \in \mathcal{F}^{i}\left(Q \cap K^{c}\right)} P\right\} .
$$

Given $P \in \mathcal{F}^{k}\left(Q \cap K^{c}\right)$, let $J_{P} \in \mathcal{P}^{k-1}$ be such that $P \subset J_{P}$. Note that $J_{P} \cap K \neq \emptyset$. Choose $y_{P} \in J_{P} \cap K$. Then

$$
B_{d}\left(z_{P}, C_{5} \lambda_{1}^{k}\right) \subset P \subset J_{P} \subset B_{d}\left(y_{P}, C_{6} \lambda_{1}^{k-1}\right) .
$$

We claim that for every $Q$

$$
\sum_{P \in \mathcal{F}^{k}\left(Q \cap K^{c}\right)} 1_{B_{d}\left(y_{P}, C_{6} \lambda_{1}^{k-1}\right)} \leq C_{42} .
$$

Here

$$
C_{42}=\frac{C_{2}^{2}\left(2 C_{6}\right)^{D}}{C_{5}^{D} \lambda_{1}^{D}}
$$

Indeed, suppose there is $y$ and $P_{1}, \ldots, P_{N} \in \mathcal{F}^{k}\left(Q \cap K^{c}\right)$ such that $y \in B_{d}\left(y_{P_{i}}, C_{6} \lambda_{1}^{k-1}\right)$, for every $i \leq N$. In particular

$$
\cup_{i} B_{d}\left(z_{P_{i}}, C_{5} \lambda_{1}^{k}\right) \subset \cup_{i} P_{i} \subset \cup_{i} J_{P_{i}} \subset B_{d}\left(y, 2 C_{6} \lambda_{1}^{k-1}\right) .
$$

so

$$
N \frac{1}{C_{2}}\left(C_{5} \lambda_{1}^{k}\right)^{D} \leq C_{2}\left(2 C_{6} \lambda_{1}^{k-1}\right)^{D}
$$


and consequently $N \leq C_{42}$. This proves the claim. Choose $q \in Q \cap K$. Then (4.34) and (4.35) implies

$$
\begin{aligned}
\sum_{P \in \mathcal{F}^{k}\left(Q \cap K^{c}\right)}|P|^{\alpha / D} & \leq C_{2} \sum_{P \in \mathcal{F}^{k}\left(Q \cap K^{c}\right)}\left|C_{6} \lambda_{1}^{k}\right|^{\alpha} \\
& \leq C_{2} \lambda_{1}^{\alpha} \sum_{P \in \mathcal{F}^{k}\left(Q \cap K^{c}\right)}\left|C_{6} \lambda_{1}^{k-1}\right|^{\alpha} \\
& \leq C_{39} C_{2} \lambda_{1}^{\alpha} \sum_{P \in \mathcal{F}^{k}\left(Q \cap K^{c}\right)} \mu\left(B_{d}\left(y_{P}, C_{6} \lambda_{1}^{k-1}\right)\right) \\
& \leq C_{42} C_{39} C_{2} \lambda_{1}^{\alpha} \mu\left(\bigcup_{P \in \mathcal{F}^{k}\left(Q \cap K^{c}\right)} B_{d}\left(y_{P}, C_{6} \lambda_{1}^{k-1}\right)\right) \\
& \leq C_{42} C_{39} C_{2} \lambda_{1}^{\alpha} \mu\left(B_{d}\left(q, 4 C_{6} \lambda_{1}^{k_{0}(Q)-1}\right)\right) \\
& \leq C_{42} C_{39}^{2} C_{2} \lambda_{1}^{\alpha}\left|4 C_{6} \lambda_{1}^{k_{0}(Q)-1}\right|^{\alpha} \\
& \leq 4{ }^{\alpha} C_{42} C_{39}^{2} C_{2} \frac{C_{6}^{\alpha}}{C_{5}^{\alpha}}\left(\left|C_{5} \lambda_{1}^{k_{0}(Q)}\right|^{D}\right)^{\alpha / D} \\
& \leq 4{ }^{\alpha} C_{42} C_{39}^{2} C_{2}^{1+\alpha / D} \frac{C_{6}^{\alpha}}{C_{5}^{\alpha}}\left(m\left(B_{d}\left(z_{Q}, C_{5} \lambda_{1}^{k_{0}(Q)}\right)\right)\right)^{\alpha / D} \\
& \leq 4{ }^{\alpha} C_{42} C_{39}^{2} C_{2}^{1+\alpha / D} \frac{C_{6}^{\alpha}}{C_{5}^{\alpha}}|Q|^{\alpha / D}
\end{aligned}
$$

Take

$$
C_{40}=4^{\alpha} C_{42} C_{39}^{2} C_{2}^{1+\alpha / D} \frac{C_{6}^{\alpha}}{C_{5}^{\alpha}} .
$$

Suppose now that every element of $\mathcal{P}$ is a connected set and let $\Omega \subset K^{c}$ be such that $\partial \Omega \subset K$. Given $Q \in \mathcal{P}$ let $\mathcal{F}^{k}(Q \cap \Omega) \subset \mathcal{F}^{k}\left(Q \cap K^{c}\right)$ be the subfamily of all $P \in \mathcal{F}^{k}\left(Q \cap K^{c}\right)$ such that $P \cap \Omega \neq \emptyset$. Since $P$ is a connected set, if $P$ is not contained in $\Omega$ we would have $\emptyset \neq P \cap \partial \Omega \subset P \cap K$, which contradicts $P \in \mathcal{F}^{k}\left(Q \cap K^{c}\right)$. So $P \subset \Omega$ and in particular

$$
Q \cap \Omega=\cup_{k \geq k_{0}(Q)} \cup_{P \in \mathcal{F}^{k}(Q \cap \Omega)} P .
$$

This easily implies that $\Omega$ is also a $\left(\alpha / D, C_{40}, C_{41}\right)$-strongly regular domain.

Remark 4.2. The theory of conformal expanding dynamical systems provides plenty of examples of strongly regular domains on $\mathbb{R}^{2}$. For instance consider the Julia set $J$ of a hyperbolic polynomial. This is a compact subset, so let $I$ be a square such that $J \subset I$. We endowed $I$ with a good grid generated by dyadic squares $\mathcal{D}$ and the Lebesgue measure $m$. The associated Besov space $\mathcal{B}_{p, q}^{s}(I, \mathcal{D})$ coincides with the Besov space of the homogenous space $(I, m)$. Then $J$ supports a geometric measure $\mu$ that satisfies the assumptions of Proposition 4.1. See Przytycki and Urbański [25] and also the survey Urbański [33]. In particular every connected component of $I \cap J^{c}$ is a strongly regular domain of the homogenous space $(I, m)$. We can consider for instance the Julia set $J$ of a quadratic polynomial $x^{2}+c$, with $c$ small (see Figure 3.) 
D. SMANIA

\section{GETTING $\mathbb{R E A L}$.}

\section{ISOMETRY With Besov SPACES DEFINED BY INTERVALS}

A case that provides a very rich class of examples is when $I=[0,1], m$ is the Lebesgue measure and the good grid consists in sequences of partitions by intervals. It turns out that in the point of view of Banach spaces up to isometries, those Besov spaces are the only ones.

Proposition 5.1. Let $\mathcal{B}_{p, q}^{s}\left(I, \mathcal{P}, \mathcal{A}_{s, p}^{s z}\right)$, with $0<s$ and $p, q \geq 1$, be a Besov-ish space defined on a probability space $(I, m)$ with a good grid $\mathcal{P}$. Then there is a good grid $\mathcal{G}$ formed by partitions by intervals of $[0,1]$ such that, considering the Lebesgue measure on I we have that the Banach space $\mathcal{B}_{p, q}^{s}\left(I, \mathcal{P}, \mathcal{A}_{s, p}^{s z}\right)$ is isometric to $\mathcal{B}_{p, q}^{s}\left([0,1], \mathcal{G}, \mathcal{A}_{s, p}^{s z}\right)$.

Proof. We can easy define a function $h$ that associated to each $P \in \mathcal{P}$ an interval $h(P) \subset[0,1]$ such that

i. $m(P)=|h(P)|$,

ii. If $P, Q \in \mathcal{P}$, with $m(P \cap Q)=0$ then $|h(P) \cap h(Q)|=0$.

iii. If $P, Q \in \mathcal{P}$ with $P \subset Q$ then $\overline{h(P)} \subset \overline{h(Q)}$.

Define $\mathcal{G}^{k}=\left\{h(P), P \in \mathcal{P}^{k}\right\}$ and

$$
T\left(\sum_{i \leq n} c_{i} 1_{P_{i}}\right)=\sum_{i \leq n} c_{i} 1_{h\left(P_{i}\right)}
$$

for every $c_{i} \in \mathbb{C}$ and $P_{i} \in \mathcal{P}$. Then $T$ is well defined and it extends to an isometry

$$
T: L^{p}(I) \rightarrow L^{p}([0,1]) .
$$

It is easy to see that for every canonical Souza's atom $a_{P}$ we have $T\left(a_{P}\right)=a_{h(P)}$. Together with Proposition 6.1 in [27] this implies that

$$
T: \mathcal{B}_{p, q}^{s}\left(I, \mathcal{P}, \mathcal{A}_{s, p}^{s z}\right) \rightarrow \mathcal{B}_{p, q}^{s}\left([0,1], \mathcal{G}, \mathcal{A}_{s, p}^{s z}\right)
$$

is well defined and an isometry.

Corollary 5.2. Let $(I, \rho, m)$ be a homogeneous space such that $I$ is compact and $m(I)=1$. Then the Besov space $B_{p, q}^{s}$, with $0<s<1 / p, p, q \geq 1$ and s small, is isomorphic to the corresponding Besov space of the homogeneous space given by $[0,1]$, the usual metric and the Lebesgue measure.

Proof. It follows from Proposition 2.3 and Proposition 2.5.

\section{QuASISYMmetric GRIDS}

In this section $I=[0,1], m$ is the Lebesgue measure and all grids consists in sequences of partitions by intervals. We say that a good grid $\mathcal{P}$ consisting on sequence of partitions of $I$ by intervals is a $C_{43}$-quasisymmetric grid if for every $k \geq 0$ and $P, Q \in \mathcal{P}^{k}$ satisfying $\bar{P} \cap \bar{Q} \neq \emptyset$ we have

$$
\frac{1}{C_{43}} \leq \frac{|Q|}{|P|} \leq C_{43}
$$


Let $Q$ be a closed interval in $[0,1]$. Define

$$
k_{0}(Q)=\min \left\{k \in \mathbb{N} \text {, there is } P \in \mathcal{P}^{k} \text { s.t. } P \subset Q\right\} .
$$

Lemma 6.1. The following holds.

A. Let $\mathcal{P}$ be a $\left(\lambda_{5}, \lambda_{6}\right)$-good grid. Then for every interval $Q=[a, b] \subset[0,1]$ there are families of intervals $\mathcal{F}_{1} \subset \mathcal{F}_{2} \subset \mathcal{P}^{k_{0}(Q)}$ such that

$$
\cup_{P \in \mathcal{F}_{i}} \bar{P}
$$

is an interval. Moreover

$$
\mathcal{F}_{1}=\left\{P \in \mathcal{P}^{k_{0}(Q)}, \bar{P} \subset \bar{Q}\right\}
$$

and

for $i=1,2$, and

$$
1 \leq \# \mathcal{F}_{i} \leq \frac{2}{\lambda_{5}}+2
$$

$$
\bigcup_{P \in \mathcal{F}_{1}} \bar{P} \subset \bar{Q} \subset \bigcup_{P \in \mathcal{F}_{2}} \bar{P}
$$

B. Suppose additionally that $\mathcal{P}$ is a $C_{43}$-quasisymmetric grid. There is $C_{44}>1$ such that for every interval $Q=[a, b] \subset[0,1]$ and every $z \in \partial Q$ and $W \in \mathcal{P}^{k_{0}(Q)}$ such that $z \in \bar{W}$ we have

$$
\frac{1}{C_{44}} \leq \frac{|Q|}{|W|} \leq C_{44}
$$

and for every $P \in \mathcal{F}_{2}$ we have

$$
\frac{1}{C_{44}} \leq \frac{|Q|}{|P|} \leq C_{44}
$$

Proof. Let

$$
\mathcal{F}_{1}=\left\{P \in \mathcal{P}^{k_{0}(Q)}, \bar{P} \subset \bar{Q}\right\}
$$

and

$$
\left[a_{1}, b_{1}\right]=\bigcup_{P \in \mathcal{F}_{1}} \bar{P}
$$

Then

$$
1 \leq \# \mathcal{F}_{1} \leq \frac{2}{\lambda_{5}}
$$

If $a_{1}=0$ then $a=0$ and we define $a_{2}=a_{1}$. Otherwise let $a_{2}<a_{1}$ be such that $\left[a_{2}, a_{1}\right] \in \mathcal{P}^{j(Q)}$. If $b_{1}=1$ then $b=1$ and we define $b_{2}=b_{1}$. Otherwise let $b_{2}$ be such that $b_{1}<b_{2}$ and $\left[b_{1}, b_{2}\right] \in \mathcal{P}^{j(Q)}$. Of course $a \in\left[a_{2}, a_{1}\right]$ and $b \in\left[b_{1}, b_{2}\right]$. Let

$$
\mathcal{F}_{2}=\mathcal{F}_{1} \cup\left\{\left[a_{2}, a_{1}\right],\left[b_{1}, b_{2}\right]\right\},
$$

with obvious adaptation if either $a_{1}=0$ or $b_{1}=1$. Furthermore if $\mathcal{P}$ is a $C_{43^{-}}$ quasisymmetric grid then for every $z \in\{a, b\}$ and $W \in \mathcal{P}^{j(Q)}$ such that $z \in \bar{W}$ we have

$C_{43}^{-\frac{2}{\lambda_{5}}-2}\left(\frac{2}{\lambda_{5}}+2\right)^{-1} \leq \frac{1}{|W|} \min \left\{|P|, P \in \mathcal{F}_{1}\right\} \leq \frac{|Q|}{|W|} \leq \frac{1}{|W|} \sum_{P \in \mathcal{F}_{2}}|P| \leq\left(\frac{2}{\lambda_{5}}+2\right) C_{43}^{\frac{2}{\lambda_{5}}+2}$,

and the same estimates holds replacing $W$ by $P \in \mathcal{F}_{2}$.

Proposition 6.2. The following holds. 
A. Let $h:[0,1] \rightarrow[0,1]$ be a quasisymmetric map and $\mathcal{D}_{N}$ be the grid of $N$-adic intervals. Then the grid $\mathcal{P}=h\left(\mathcal{D}_{N}\right)$ defined by

$$
\mathcal{P}^{k}=\left\{h(P), P \in \mathcal{D}_{N}^{k}\right\}
$$

is a quasisymmetric grid.

B. On the other hand if $\mathcal{P}$ is a quasisymmetric grid such that every $P \in \mathcal{P}$ has exactly $N$ children then there is a quasisymmetric function $h$ such that $\mathcal{P}=h\left(\mathcal{D}_{N}\right)$.

Proof of $A$. If $h$ is a quasisymmetric map then there is $C_{43}>0$ such that (6.36) holds for every two intervals $P=[x-\delta, x], Q=[x, x+\delta]$ with $\{x-\delta, x+\delta\} \subset[0,1]$. In particular $h\left(\mathcal{D}_{N}\right)$ is a quasisymmetric grid.

Proof of $B$. Define $\phi:[0,1] \rightarrow[0,1]$ as

$$
\phi(x)=\lim _{k} \frac{1}{N^{k}} \#\left\{P \in \mathcal{P}^{k}, P \subset[0, x]\right\} .
$$

the function $\phi$ is well-defined since the sequence in the r.h.s. is non-decreasing and bounded by 1 . It is easy to see that $\phi$ is monotone increasing and continuous, so it is a homeomorphism. Let $L=[x-\delta, x], R=[x, x+\delta]$ with $\{x-\delta, x+\delta\} \subset[0,1]$. It follows from Lemma 6.1.A. that

$$
\frac{1}{N^{j(L)}} \leq \phi(x)-\phi(x-\delta) \leq \frac{1}{N^{j(L)}}\left(\frac{2}{\lambda_{5}}+2\right)
$$

and

$$
\frac{1}{N^{j(R)}} \leq \phi(x+\delta)-\phi(x) \leq \frac{1}{N^{j(R)}}\left(\frac{2}{\lambda_{5}}+2\right) .
$$

Let $W_{L} \in \mathcal{P}^{j(L)}, W_{R} \in \mathcal{P}^{j(R)}$ be such that $x \in \bar{W}_{R} \cap \bar{W}_{L}$. Due Lemma 6.1.B we have

so

$$
C_{44}^{-1} \leq \frac{\delta}{\left|W_{R}\right|} \leq C_{44}, C_{44}^{-1} \leq \frac{\delta}{\left|W_{L}\right|} \leq C_{44},
$$

$$
C_{44}^{-2} \leq \min \left\{\frac{\left|W_{L}\right|}{\left|W_{R}\right|}, \frac{\left|W_{R}\right|}{\left|W_{L}\right|}\right\} .
$$

On the other hand since $\mathcal{P}$ is a quasisymmetric (and in particular good) grid we have

$$
\min \left\{\frac{\left|W_{L}\right|}{\left|W_{R}\right|}, \frac{\left|W_{R}\right|}{\left|W_{L}\right|}\right\} \leq C_{43} \lambda_{6}^{|j(L)-j(R)|}
$$

so $|j(L)-j(R)| \leq C_{45}$, where $C_{45}$ does not depend on $x$ and $\delta$. By (6.37) and

$$
N^{-C_{45}}\left(\frac{2}{\lambda_{5}}+2\right)^{-1} \leq \frac{|\phi(x)-\phi(x-\delta)|}{|\phi(x+\delta)-\phi(x)|} \leq N^{C_{45}}\left(\frac{2}{\lambda_{5}}+2\right),
$$

so $\phi$ is a quasisymmetric map and consequently the same holds for $h=\phi^{-1}$.

Proposition 6.3. Let $\mathcal{P}$ be a quasisymmetric $\left(\lambda_{5}, \lambda_{6}\right)$-good grid and $Q \subset[0,1]$ be an interval. There are families of intervals $\mathcal{F}^{k}(Q), k \in \mathbb{Z}$, and increasing sequences $j_{i}^{+}, j_{i}^{-} \in \mathbb{N}$ such that

A. We have that

$$
\cup_{k \in \mathbb{Z}} \mathcal{F}^{k}(Q)
$$

is a countable partition of $Q$. 
B. We have

$$
\mathcal{F}^{i}(Q) \subset \mathcal{P}^{j_{i}^{+}}, \mathcal{F}^{-i}(Q) \subset \mathcal{P}^{j_{i}^{-}}
$$

with

$$
j_{0}^{+}=j_{0}^{-}=\min \left\{j \geq 0 \text { s.t. there is } P \in \mathcal{P}^{j} \text { satisfying } \bar{P} \subset Q\right\} \text {. }
$$

C. There exists $\theta \in[0,1)$ and $r_{0}$ such that

$$
|S| \leq C_{43}^{2 / \lambda_{5}+1} \lambda_{6}^{r}|W|
$$

for every $S \in \mathcal{F}^{-i-r}(Q), W \in \mathcal{F}^{-i}(Q), i \geq 0$, and for every $S \in \mathcal{F}^{i+r}(Q)$, $W \in \mathcal{F}^{i}(Q), i \geq 0$.

D. We have

$$
1 \leq \# \mathcal{F}^{k}(Q) \leq \frac{2}{\lambda_{5}}
$$

Proof. Let $Q=[a, b]$ and

$$
j_{0}=\min \left\{j \geq 0 \text { s.t. there is } P \in \mathcal{P}^{j} \text { satisfying } \bar{P} \subset Q\right\} .
$$

We define families $\mathcal{F}_{\star}^{k}(Q)$ with $k \in \mathbb{Z}$, in the following way. Let

$$
\mathcal{F}^{0}(Q)=\left\{P \in \mathcal{P}^{j_{0}} \text { satisfying } \bar{P} \subset Q\right\}
$$

and

$$
\left[a_{0}, b_{0}\right]=\overline{\cup_{P \in \mathcal{F}^{0}(Q)} P} .
$$

Let $j_{0}^{+}=j_{0}^{-}=j_{0}$. By induction on $i \geq 0$ define $j_{i}^{-}$as the smallest $j>j_{i-1}^{-}$such that

$$
\mathcal{F}^{-i}(Q)=\left\{P \in \mathcal{P}^{j} \text { satisfying } \bar{P} \subset\left[a, a_{i-1}\right]\right\} \neq \emptyset,
$$

and $j_{i}^{+}$as the smallest $j>j_{i-1}^{+}$such that

$$
\mathcal{F}^{i}(Q)=\left\{P \in \mathcal{P}^{j} \text { satisfying } \bar{P} \subset\left[b_{i-1}, b\right]\right\} \neq \emptyset
$$

and

$$
\left[a_{i}, b_{i}\right]=\left(\cup_{0 \leq k \leq i} \cup_{P \in \mathcal{F}^{k}(Q)} \bar{P}\right) \bigcup\left(\cup_{0 \leq k \leq i} \cup_{P \in \mathcal{F}^{-k}(Q)} \bar{P}\right) .
$$

Note that

for every $k \in \mathbb{Z}$ and

$$
1 \leq \# \mathcal{F}^{k}(Q) \leq \frac{2}{\lambda_{5}}
$$

is a countable partition of $Q$.

$$
\cup_{k \in \mathbb{Z}} \mathcal{F}^{k}(Q)
$$

Let $P \in \mathcal{F}^{-i}(Q)$ be such that $a_{i} \in \partial P$. Let $P^{\prime} \in \mathcal{P}^{j_{i}^{-}}$be such that $P^{\prime} \subset\left[0, a_{i}\right]$ and $\overline{P^{\prime}} \cap P \neq \emptyset$. Since $P^{\prime} \notin \mathcal{F}^{-i}(Q)$ we have that

$$
\left[a, a_{i}\right] \subset P^{\prime}
$$

Moreover since $\mathcal{P}$ is a quasymmetric grid we have $\left|P^{\prime}\right| \leq C_{43}|P|$ and $\left|P^{\prime}\right| \leq$ $C_{43}^{2 / \lambda_{5}+1}|W|$ for every $W \in \mathcal{F}^{-i}(Q)$. In particular

$$
\left|a_{i+1}-a_{i}\right| \leq\left|a-a_{i}\right| \leq C_{43}\left|a_{i}-a_{i-1}\right|,
$$

and for every $S \in \mathcal{P}^{j_{i}^{+}+r}$ such that $S \subset\left[a, a_{i}\right]$ we have

$$
|S| \leq \lambda_{6}^{r} C_{43}^{2 / \lambda_{5}+1}|W|
$$

for every $W \in \mathcal{F}^{-i}(Q)$. We can use the same argument replacing $a$ by $b$ and $a_{i}$ by $b_{i}$ in the above argument. So C. holds. 


\section{7. Ехотіс $\mathcal{B}_{p, q}^{s}$ FOR $p \neq q$}

The goal of this section is to show how sensitive is the dependence of $\mathcal{B}_{p, q}^{s}$, for $p \neq q$, with respect to the grid $\mathcal{P}$. Choosing distinct grids in a familiar space as $([0,1], m)$, where $m$ is the Lebesgue measure, may give origin to distinct Besov-ish spaces. The reader should compare this result with Bourdaud and Sickel [6] and Bourdaud [5]

Proposition 7.1. Let $I=[0,1], m$ is the Lebesgue measure, and $\mathcal{P}_{\star}$ and $\mathcal{P}_{\circ}$ be quasisymmetric grids such that for every $P \in \mathcal{P}_{i}^{k}, i=\star, \circ$ and $k \geq 0$ we have

$$
\#\left\{Q \in \mathcal{P}_{i}^{k+1} \text { s.t. } Q \subset P\right\}=2 .
$$

Let $h_{i}:[0,1] \rightarrow[0,1]$ be a quasisymmetric map such that $h_{i}\left(\mathcal{P}_{i}\right)=\mathcal{D}$. The following statements are equivalent.

A. We have $\mathcal{B}_{p, q}^{s}\left(\mathcal{P}_{\circ}\right) \subset \mathcal{B}_{p, q}^{s}\left(\mathcal{P}_{\star}\right)$.

B. We have $\mathcal{B}_{p, q}^{s}\left(\mathcal{P}_{\circ}\right)=\mathcal{B}_{p, q}^{s}\left(\mathcal{P}_{\star}\right)$.

C. The map $h_{\star} \circ h_{\circ}^{-1}$ is a bi-Lipchitz function.

Proof. Without loss of generality we assume that $\mathcal{P}_{\star}$ and $\mathcal{P}_{\circ}$ are $\left(\lambda_{5}, \lambda_{6}\right)$-good grids. For an interval $Q \subset[0,1]$ define

$$
\begin{aligned}
& j_{0}^{\star}(Q)=\min \left\{j \geq 0 \text { s.t. there is } P \in \mathcal{P}_{\star}^{j} \text { satisfying } \bar{P} \subset Q\right\} . \\
& j_{0}^{\circ}(Q)=\min \left\{j \geq 0 \text { s.t. there is } P \in \mathcal{P}_{\circ}^{j} \text { satisfying } \bar{P} \subset Q\right\} . \\
& j_{0}^{D}(Q)=\min \left\{j \geq 0 \text { s.t. there is } P \in \mathcal{D}_{\circ}^{j} \text { satisfying } \bar{P} \subset Q\right\} .
\end{aligned}
$$

We claim that

Claim 1. There is $C_{46} \geq 1$ such that for every $Q \in \mathcal{P}_{\circ}^{k}$ and $P \in \mathcal{P}_{\star}^{j_{0}^{\star}(Q)}$ satisfying $P \subset Q$ we have $k \leq j_{0}^{\circ}(P) \leq k+C_{46}$.

and

Claim 2. There is $C_{47} \geq 0$ such that for every $Q_{1}, Q_{2} \in \mathcal{P}_{\circ}^{k}$, with $k \in \mathbb{N}$, and satisfying $\bar{Q}_{1} \cap \bar{Q}_{2} \neq \emptyset$ we have

$$
\left|j_{0}^{\star}\left(Q_{1}\right)-j_{0}^{\star}\left(Q_{2}\right)\right| \leq C_{47} .
$$

Both Claims 1 and 2 follows easily from Lemma 6.1.

Claim 3. Suppose that

$$
C_{48}=\sup _{k} \#\left\{j_{0}^{\star}(Q), Q \in \mathcal{P}_{\circ}^{k}\right\}<\infty .
$$

Then $h_{\star}^{-1} \circ h_{\circ}$ is a bi-Lipchitz function.

Indeed if

$$
M_{k}=\max \left\{j_{0}^{\star}(Q), Q \in \mathcal{P}_{\circ}^{k}\right\} \text { and } m_{k}=\min \left\{j_{0}^{\star}(Q), Q \in \mathcal{P}_{\circ}^{k}\right\}
$$

then Claim 2. implies that

$$
\sup _{k}\left(M_{k}-m_{k}\right) \leq C_{48}\left(C_{47}+1\right) .
$$

For every $Q \in \mathcal{P}_{\circ}$ choose $P_{Q} \in \mathcal{P}^{j_{\star}(Q)}$ such that $P_{Q} \subset Q$. Of course

$$
\#\left\{P_{Q}, Q \in \mathcal{P}_{\circ}^{k}\right\}=2^{k}
$$


and

$$
\left\{P_{Q}, Q \in \mathcal{P}_{\circ}^{k}\right\} \subset \cup_{m_{k} \leq j \leq M_{k}} \mathcal{P}_{\star}^{j},
$$

so

$$
2^{k} \leq \sum_{m_{k} \leq j \leq M_{k}} 2^{j} \leq 2^{M_{k}+1} \leq 2^{C_{47}+1} 2^{m_{k}} .
$$

By Lemma 6.1.A each $Q \in \mathcal{P}_{\circ}^{k}$ intersects at most $1 / \lambda_{5}+2$ intervals in $\mathcal{P}_{\star}^{j_{\star}(Q)}$, so $Q$ intersects at most $2^{C_{47}}\left(1 / \lambda_{5}+2\right)$ intervals in $\mathcal{P}_{\star}^{M_{k}}$. Since $\mathcal{P}_{\circ}^{k}$ covers $[0,1]$ we have

$$
\mathcal{P}_{\star}^{M_{k}}=\bigcup_{Q \in \mathcal{P}_{\circ}^{k}}\left\{P \in \mathcal{P}_{\star}^{M_{k}} \text { s.t. } P \cap Q \neq \emptyset\right\},
$$

so

$$
2^{m_{k}} \leq 2^{M_{k}} \leq 2^{C_{47}}\left(1 / \lambda_{5}+2\right) 2^{k},
$$

so there are constants $A, B$ such that

$$
k+A \leq m_{k} \leq k+B .
$$

Note that for every interval $W \subset[0,1]$ with $k=j_{0}^{D}(W)$ we have

$$
2^{-k} \leq|W| \leq 2^{-k+1},
$$

so $\left.k=j_{0}^{\circ}\left(h_{\circ}^{-1} W\right)\right)$ and consequently $j_{0}^{D}\left(h_{\star} \circ h_{\circ}^{-1}(W)\right)=j_{0}^{\star}\left(h_{\circ}^{-1}(W)\right) \in\left[m_{k}, M_{k}\right]$.

We conclude that

$\frac{1}{C_{49}}|W| \leq 2^{-k-B+C_{48}\left(C_{47}+1\right)} \leq 2^{-M_{k}} \leq\left|h_{\star} \circ h_{\circ}^{-1}(W)\right| \leq 2^{-m_{k}+1} \leq 2^{-k-A+1} \leq C_{49}|W|$,

so $h_{\star} \circ h_{\circ}^{-1}$ is bi-Lipchitz. This proves Claim 3 .

Claim 4. If $h_{\star} \circ h_{\circ}^{-1}$ is a bi-Lipchitz function then $\mathcal{B}_{p, q}^{s}\left(\mathcal{P}_{\circ}\right)=\mathcal{B}_{p, q}^{s}\left(\mathcal{P}_{\star}\right)$.

Let $Q \in \mathcal{P}_{\circ}^{i}$. Then

$$
2^{-i} \leq\left|h_{\circ}(Q)\right| \leq 2^{-i+1}
$$

and consequently

so

$$
\frac{1}{C} 2^{-i} \leq\left|h_{\star}(Q)\right| \leq C 2^{-i+1}
$$

$$
i+A \leq j_{0}^{\star}(Q) \leq i+B
$$

for some $A, B$ that does not depend on $i$. Let $k_{i}=i+B$. Consider Proposition 6.3 taking $\mathcal{P}=\mathcal{P}_{\star}$ and let

$$
\mathcal{G}^{k}(Q)=\left\{P \in \mathcal{F}^{\ell}(Q), \ell \geq 0, j_{\ell}^{+}=k\right\} \cup\left\{P \in \mathcal{F}^{\ell}(Q), \ell<0, j_{|\ell|}^{-}=k\right\} .
$$

Then by Lemma 6.1.B we have

$$
a_{Q}=\sum_{k \geq j_{0}^{\star}(Q)} \sum_{P \in \mathcal{G}^{k}(Q)}\left(\frac{|P|}{|Q|}\right)^{1 / p-s} a_{P}
$$

with

$$
\sum_{\mathcal{G}^{k}(Q)}\left(\frac{|P|}{|Q|}\right)^{1-s p} \leq \frac{4 C_{44}^{1-s p}}{\lambda_{5}} \lambda_{6}^{\left(k-k_{i}\right)(1-s p)} .
$$

By Proposition 8.1.C in [27] we have $\mathcal{B}_{p, q}^{s}\left(\mathcal{P}_{\circ}\right) \subset \mathcal{B}_{p, q}^{s}\left(\mathcal{P}_{\star}\right)$. The proof of the reverse inclusion is similar. 
Claim 5. Suppose that $p>q$ and

$$
\sup _{k} \#\left\{j_{0}^{\star}(Q), Q \in \mathcal{P}_{\circ}^{k}\right\}=+\infty .
$$

Then there is $f \in \mathcal{B}_{p, q}^{s}\left(\mathcal{P}_{\circ}\right) \backslash \mathcal{B}_{p, q}^{s}\left(\mathcal{P}_{\star}\right)$.

Step $I$. Indeed this implies that for every $n \in \mathbb{N}$ we can find $r_{n}, v_{n} \in \mathbb{N}$ and families with $r_{n}$ elements $\left\{Q_{1}^{n}, \ldots, Q_{r_{n}}^{n}\right\} \subset \mathcal{P}_{\circ}^{v_{n}}$, with $\lim _{n} r_{n}=+\infty$ and

$$
\left|j_{0}^{\star}\left(Q_{i}^{n}\right)-j_{0}^{\star}\left(Q_{\ell}^{n}\right)\right| \geq 3 C_{46}
$$

for every $i \neq \ell$. Taking a subsequence we can also assume that $v_{n}$ is increasing, $v_{n} \geq r_{n}$,

for every $n<m$, and

$$
j_{0}^{\star}\left(Q_{i}^{n}\right)+3 C_{46}<j_{0}^{\star}\left(Q_{\ell}^{m}\right)
$$

$$
\sum_{1 \leq i \leq r_{n}} \frac{1}{i}>2^{n q}
$$

For every $n$ and $1 \leq i \leq r_{n}$ choose $P_{i}^{n} \in \mathcal{P}_{\star}^{j_{0}^{\star}\left(Q_{i}^{n}\right)}$ such that $P_{i}^{n} \subset Q_{i}^{n}$. Note that Claim 1 implies $v_{n} \leq j_{0}^{\circ}\left(P_{i}^{n}\right) \leq v_{n}+C_{46}$.

Step II. For every $n$ and $i \leq r_{n}$ let $A_{i}^{n}, B_{i}^{n}$ be the only children of $P_{i}^{n}$. Define $S_{i}^{n}=\left(A_{i}^{n}, B_{i}^{n}\right)$ and let $\phi_{S_{i}^{n}}$ be the corresponding element of the unconditional basis in $L^{p}$ (note that in Claim 5 we have $p>1$ ) defined in Section 14 in [27] using the grid $\mathcal{P}_{\star}$. Then

$$
f=\sum_{n} \sum_{i \leq r_{n}} \frac{1}{2^{n} i^{1 / q}\left|P_{i}^{n}\right|^{1 / p-s-1 / 2}} \phi_{S_{i}^{n}}
$$

is the Haar representation of a function in $L^{p}$. Indeed we have $P_{i}^{n} \cap P_{j}^{n}=\emptyset$ for $i \neq j$, so

$$
\begin{aligned}
& \left|\sum_{i \leq r_{n}} \frac{1}{2^{n} i^{1 / q}\left|P_{i}^{n}\right|^{1 / p-s-1 / 2}} \phi_{S_{i}^{n}}\right|_{p}^{p} \\
= & \frac{1}{2^{n p}} \sum_{i \leq r_{n}}\left|\frac{1}{i^{1 / q}\left|P_{i}^{n}\right|^{1 / p-s-1 / 2}} \phi_{S_{i}^{n}}\right|_{p}^{p} \\
= & \frac{1}{2^{n p}} \sum_{i \leq r_{n}} \frac{\left|P_{i}^{n}\right|^{s p}}{i^{p / q}} \leq \frac{1}{2^{n p}} \sum_{i=1}^{\infty} \frac{1}{i^{p / q}} \leq \frac{C_{50}}{2^{n p}} .
\end{aligned}
$$

So

$$
\begin{aligned}
& \left|\sum_{j} \sum_{j_{0}^{\star}\left(Q_{i}^{n}\right)=j} \frac{1}{2^{n} i^{1 / q}\left|P_{i}^{n}\right|^{1 / p-s-1 / 2}} \phi_{S_{i}^{n}}\right|_{p} \\
= & \left|\sum_{n} \sum_{i \leq r_{n}} \frac{1}{2^{n} i^{1 / q}\left|P_{i}^{n}\right|^{1 / p-s-1 / 2}} \phi_{S_{i}^{n}}\right|_{p} \\
\leq & 2 C_{50}^{1 / p} .
\end{aligned}
$$

We conclude that $f \in L^{p}$, with $p>q \geq 1$.

Step III. Since, the ordering of the sum in (7.44) does not matter, so 


$$
f=\sum_{j} \sum_{j_{0}^{\star}\left(Q_{i}^{n}\right)=j} \frac{1}{2^{n} i^{1 / q}\left|P_{i}^{n}\right|^{1 / p-s-1 / 2}} \phi_{S_{i}^{n}}
$$

Since $j_{0}^{\star}\left(P_{i}^{n}\right) \neq j_{0}^{\star}\left(P_{\ell}^{m}\right)$ provided $(n, i) \neq(m, \ell)$ we have

$$
\begin{aligned}
N_{\text {haar }}^{\star}(f) & =\left(\sum_{j \geq 1}\left(\sum_{P_{i}^{n} \in \mathcal{P}_{\star}^{j}} \frac{1}{2^{n p} i^{p / q}}\right)^{q / p}\right)^{1 / q}, \\
& =\left(\sum_{n} \sum_{i \leq r_{n}} \frac{1}{2^{n q} i}\right)^{1 / q} \\
& =\left(\sum_{n} \frac{1}{2^{n q}} \sum_{i \leq r_{n}} \frac{1}{i}\right)^{1 / q}=+\infty,
\end{aligned}
$$

so $f \notin \mathcal{B}_{p, q}^{s}\left(\mathcal{P}_{\star}\right)$.

Step IV. On the other hand, note that

$$
f=\sum_{j} \sum_{j_{0}^{\circ}\left(Q_{i}^{n}\right)=j} c_{Q_{i}^{n}} h_{Q_{i}^{n}}=\sum_{n} \sum_{i \leq r_{n}} c_{Q_{i}^{n}} h_{Q_{i}^{n}},
$$

where

and

$$
c_{Q_{i}^{n}}=\frac{1}{2^{n} i^{1 / q}}
$$

with

$$
\begin{aligned}
& t_{i}^{n}=\frac{1}{\left|P_{i}^{n}\right|^{1 / p-s-1 / 2}}\left(\frac{1}{\left|A_{i}^{n}\right|}+\frac{1}{\left|B_{i}^{n}\right|}\right)^{-1 / 2}\left|A_{i}^{n}\right|^{1 / p-s-1} \leq C_{51}, \\
& z_{i}^{n}=\frac{1}{\left|P_{i}^{n}\right|^{1 / p-s-1 / 2}}\left(\frac{1}{\left|A_{i}^{n}\right|}+\frac{1}{\left|B_{i}^{n}\right|}\right)^{-1 / 2}\left|B_{i}^{n}\right|^{1 / p-s-1} \leq C_{51} .
\end{aligned}
$$

Here $C_{51}$ depends only on the geometry of $\mathcal{P}_{\star}, s$ and $p$. We have

$$
\begin{aligned}
\left(\sum_{j}\left(\sum_{j_{0}^{\circ}\left(Q_{i}^{n}\right)=j} c_{Q_{i}^{n}}^{p}\right)^{q / p}\right)^{1 / q} & =\left(\sum_{n} \frac{1}{2^{n q}}\left(\sum_{i \leq r_{n}} \frac{1}{i^{p / q}}\right)^{q / p}\right)^{1 / q} \\
& =\left(\sum_{n} \frac{1}{2^{n q}}\right)^{1 / q}\left(\sum_{i} \frac{1}{i^{p / q}}\right)^{1 / p}<\infty .
\end{aligned}
$$

For every $k \in \mathbb{N}$ define

$$
\mathcal{Y}^{k}\left(Q_{i}^{n}\right)=\mathcal{P}_{\circ}^{k} \cap \bigcup_{\ell}\left(\mathcal{F}^{\ell}\left(A_{i}^{n}\right) \cup \mathcal{F}^{-\ell}\left(A_{i}^{n}\right) \cup \mathcal{F}^{\ell}\left(B_{i}^{n}\right) \cup \mathcal{F}^{-\ell}\left(B_{i}^{n}\right)\right),
$$

where the families $\mathcal{F}^{\ell}$ are as defined in Proposition 6.3 taking $\mathcal{P}=\mathcal{P}_{\mathrm{o}}$. Of course $\mathcal{Y}^{k}\left(Q_{i}^{n}\right)=\emptyset$ for $k<v_{n}$, and for every $P \in \mathcal{Y}^{k}\left(Q_{i}^{n}\right)$ we have $P \subset Q_{i}^{n}$, and consequently

$$
|P| \leq \lambda_{6}^{k-v_{n}}\left|Q_{i}^{n}\right| \leq C_{44} \lambda_{5}^{-C_{46}} \lambda_{6}^{k-v_{n}} \min \left\{\left|A_{i}^{n}\right|,\left|B_{i}^{n}\right|\right\} .
$$

Moreover by Proposition 6.3.D

$$
\# \mathcal{Y}^{k}\left(Q_{i}^{n}\right) \leq \frac{8}{\lambda_{5}}
$$


Then

$$
\begin{aligned}
h_{Q_{i}^{n}}= & t_{i}^{n} \frac{1_{A_{i}^{n}}}{\left|A_{i}^{n}\right|^{1 / p-s}}-z_{i}^{n} \frac{1_{B_{i}^{n}}}{\left|B_{i}^{n}\right|^{1 / p-s}} \\
& =\sum_{k}\left[\sum_{\substack{P \in \mathcal{Y}^{k}\left(Q_{i}^{n}\right) \\
P \subset A_{i}^{n}}} t_{i}^{n}\left(\frac{|P|}{\left|A_{i}^{n}\right|}\right)^{1 / p-s} a_{P}\right]-\left[\sum_{\substack{P \in \mathcal{Y}^{k}\left(Q_{i}^{n}\right) \\
P \subset B_{i}^{n}}} z_{i}^{n}\left(\frac{|P|}{\left|B_{i}^{n}\right|}\right)^{1 / p-s} a_{P}\right] \\
& =\sum_{k} \sum_{P \in \mathcal{Y}^{k}\left(Q_{i}^{n}\right)} s_{P, Q_{i}^{n}} a_{P}
\end{aligned}
$$

Here $a_{W}$ is the canonical Souza's atom on $W$ and

$$
s_{P, Q_{i}^{n}}=t_{i}^{n}\left(\frac{\left|P \cap A_{i}^{n}\right|}{\left|A_{i}^{n}\right|}\right)^{1 / p-s}-z_{i}^{n}\left(\frac{\left|P \cap B_{i}^{n}\right|}{\left|B_{i}^{n}\right|}\right)^{1 / p-s} .
$$

Consequently

$$
\begin{aligned}
& \sum_{P \in \mathcal{Y}^{k}\left(Q_{i}^{n}\right)}\left|s_{P, Q_{i}^{n}}\right|^{p} \\
\leq & 2^{p}\left[\sum_{\substack{P \in \mathcal{Y}^{k}\left(Q_{i n}^{n}\right) \\
P \subset A_{i}^{n}}}\left(t_{i}^{n}\right)^{p}\left(\frac{|P|}{\left|A_{i}^{n}\right|}\right)^{1-s p}\right]+2^{p}\left[\sum_{\substack{P \in \mathcal{Y}^{k}\left(Q_{i}^{n}\right) \\
P \subset B_{i}^{n}}}\left(z_{i}^{n}\right)^{p}\left(\frac{|P|}{\left|B_{i}^{n}\right|}\right)^{1-s p}\right] \\
\leq & C_{52} \lambda_{6}^{\left(k-v_{n}\right)(1-s p) .}
\end{aligned}
$$

So by Proposition 8.1.A in [27](take $\mathcal{G}=\mathcal{W}=\mathcal{P}_{\mathrm{o}}, A=B=0$ and $k_{i}=i$ ) we have that $f \in \mathcal{B}_{p, q}^{s}\left(\mathcal{P}_{\circ}\right)$. This completes the proof of Claim 5 .

Claim 6. Suppose that $q>p$ and

$$
\sup _{k} \#\left\{j_{0}^{\star}(Q), Q \in \mathcal{P}_{\circ}^{k}\right\}=+\infty .
$$

Then there is $f \in \mathcal{B}_{p, q}^{s}\left(\mathcal{P}_{\star}\right) \backslash \mathcal{B}_{p, q}^{s}\left(\mathcal{P}_{\circ}\right)$.

The proof of Claim 6. has many similarities with the proof of Claim 5., however there are modifications that may not seems obvious to the reader. We describe them below.

Step I. Consider families $\left\{Q_{1}^{n}, \ldots, Q_{r_{n}}^{n}\right\} \subset \mathcal{P}_{\circ}^{v_{n}}$ as in the Step I. in the proof of Claim 5, except that we replace condition (7.43) by

$$
\sum_{1 \leq i \leq r_{n}} \frac{1}{i}>2^{n p}
$$

and we also demand $v_{n+1}>v_{n}+3 C_{46}+2$. For every $n$ and $1 \leq i \leq r_{n}$ choose $P_{i}^{n} \in \mathcal{P}_{\star}^{j_{0}^{\star}\left(Q_{i}^{n}\right)}$ such that $P_{i}^{n} \subset Q_{i}^{n}$, and also $\hat{Q}_{i}^{n} \subset P_{i}^{n}$ satisfying $\hat{Q}_{i}^{n} \in \mathcal{P}_{0}^{j_{0}^{\circ}\left(P_{i}^{n}\right)}$. We have $v_{n} \leq j_{0}^{\circ}\left(P_{i}^{n}\right) \leq v_{n}+C_{46}$.

Let $A_{i}^{n}, B_{i}^{n} \in \mathcal{P}_{\circ}^{j_{0}^{\circ}\left(P_{i}^{n}\right)+1}$ be the only children of $\hat{Q}_{i}^{n}$. Define $S_{i}^{n}=\left(A_{i}^{n}, B_{i}^{n}\right)$ and let $\phi_{S_{i}^{n}}$ be the corresponding element of the unconditional basis of $L^{t}$, for every $1<t<\infty$, defined in Section 14 in [27] using the grid $\mathcal{P}_{\mathrm{o}}$. 
Step II. Define

$$
f=\sum_{j} \sum_{j_{0}^{\star}\left(P_{i}^{n}\right)=j} \frac{1}{2^{n} i^{1 / p}\left|\hat{Q}_{i}^{n}\right|^{1 / p-s-1 / 2}} \phi_{S_{i}^{n}}
$$

We claim that $f$ is well defined and it belongs to $\mathcal{B}_{p, q}^{s}\left(\mathcal{P}_{\star}\right)$. Indeed, we can write

$$
f=\sum_{j} \sum_{j_{0}^{\star}\left(P_{i}^{n}\right)=j} c_{P_{i}^{n}} h_{P_{i}^{n}}
$$

where

$$
c_{P_{i}^{n}}=\frac{1}{2^{n} i^{1 / p}}
$$

and

$$
h_{P_{i}^{n}}=t_{i}^{n} \frac{1_{A_{i}^{n}}}{\left|A_{i}^{n}\right|^{1 / p-s}}-z_{i}^{n} \frac{1_{B_{i}^{n}}}{\left|B_{i}^{n}\right|^{1 / p-s}},
$$

with

$$
\begin{aligned}
t_{i}^{n} & =\frac{1}{\left|\hat{Q}_{i}^{n}\right|^{1 / p-s-1 / 2}}\left(\frac{1}{\left|A_{i}^{n}\right|}+\frac{1}{\left|B_{i}^{n}\right|}\right)^{-1 / 2}\left|A_{i}^{n}\right|^{1 / p-s-1} \leq C_{53}, \\
z_{i}^{n} & =\frac{1}{\left|\hat{Q}_{i}^{n}\right|^{1 / p-s-1 / 2}}\left(\frac{1}{\left|A_{i}^{n}\right|}+\frac{1}{\left|B_{i}^{n}\right|}\right)^{-1 / 2}\left|B_{i}^{n}\right|^{1 / p-s-1} \leq C_{53} .
\end{aligned}
$$

So

$$
\begin{aligned}
\left(\sum_{j}\left(\sum_{j_{0}^{\star}\left(P_{i}^{n}\right)=j} c_{\hat{Q}_{i}^{n}}^{p}\right)^{q / p}\right)^{1 / q} & =\left(\sum_{n} \sum_{i \leq r_{n}} \frac{1}{2^{n q} i^{q / p}}\right)^{1 / q} \\
& =\left(\sum_{n} \frac{1}{2^{n q}}\right)^{1 / q}\left(\sum_{i} \frac{1}{i^{q / p}}\right)^{1 / q}<\infty .
\end{aligned}
$$

For every $k \in \mathbb{N}$ define

$$
\mathcal{Y}^{k}\left(P_{i}^{n}\right)=\mathcal{P}_{\star}^{k} \cap \bigcup_{\ell} \mathcal{F}^{\ell}\left(A_{i}^{n}\right) \cup \mathcal{F}^{-\ell}\left(A_{i}^{n}\right) \cup \mathcal{F}^{\ell}\left(B_{i}^{n}\right) \cup \mathcal{F}^{-\ell}\left(B_{i}^{n}\right),
$$

where the families $\mathcal{F}^{\ell}$ are as defined in Proposition 6.3 taking $\mathcal{P}=\mathcal{P}_{\star}$. Of course $\mathcal{Y}^{k}\left(P_{i}^{n}\right)=\emptyset$ for $k<j_{0}^{\star}\left(\hat{Q}_{i}^{n}\right) \leq j_{0}^{\star}\left(P_{i}^{n}\right)$, and for every $P \in \mathcal{Y}^{k}\left(P_{i}^{n}\right)$ we have $P \subset$ $\hat{Q}_{i}^{n} \subset P_{i}^{n}$, and consequently due Proposition 6.1.B

$$
|P| \leq \lambda_{6}^{k-j_{0}^{\star}\left(P_{i}^{n}\right)}\left|P_{i}^{n}\right| \leq C_{44} \lambda_{6}^{k-j_{0}^{\star}\left(P_{i}^{n}\right)}\left|\hat{Q}_{i}^{n}\right| \leq C_{44} \lambda_{5}^{-1} \lambda_{6}^{k-j_{0}^{\star}\left(P_{i}^{n}\right)} \min \left\{\left|A_{i}^{n}\right|,\left|B_{i}^{n}\right|\right\} .
$$

\section{Moreover}

$$
\# \mathcal{Y}^{k}\left(P_{i}^{n}\right) \leq \frac{8}{\lambda_{5}}
$$

Then

$$
\begin{aligned}
& h_{P_{i}^{n}}=t_{i}^{n} \frac{1_{A_{i}^{n}}}{\left|A_{i}^{n}\right|^{1 / p-s}}-z_{i}^{n} \frac{1_{B_{i}^{n}}}{\left|B_{i}^{n}\right|^{1 / p-s}} \\
& =\sum_{k \in \mathbb{N}}\left[\sum_{\substack{P \in \mathcal{Y}^{k}\left(P_{i}^{n}\right) \\
P \subset A_{i}^{n^{n}}}} t_{i}^{n}\left(\frac{|P|}{\left|A_{i}^{n}\right|}\right)^{1 / p-s} a_{P}\right]-\left[\sum_{\substack{P \in \mathcal{Y}^{k}\left(P_{i}^{n}\right) \\
P \subset B_{i}^{n^{n}}}} z_{i}^{n}\left(\frac{|P|}{\left|B_{i}^{n}\right|}\right)^{1 / p-s} a_{P}\right] \\
& =\sum_{k \in \mathbb{N}} \sum_{P \in \mathcal{Y}^{k}\left(P_{i}^{n}\right)} s_{P, P_{i}^{n}} a_{P}
\end{aligned}
$$


Here $a_{W}$ is the canonical Souza's atom on $W$ and

$$
s_{P, P_{i}^{n}}=t_{i}^{n}\left(\frac{\left|P \cap A_{i}^{n}\right|}{\left|A_{i}^{n}\right|}\right)^{1 / p-s}-z_{i}^{n}\left(\frac{\left|P \cap B_{i}^{n}\right|}{\left|B_{i}^{n}\right|}\right)^{1 / p-s} .
$$

Consequently

$$
\begin{aligned}
& \sum_{P \in \mathcal{Y}^{k}\left(P_{i}^{n}\right)}\left|s_{P, P_{i}^{n}}\right|^{p} \\
\leq & 2^{p}\left[\sum_{\substack{P \in \mathcal{Y}^{k}\left(P_{i}^{n}\right) \\
P \subset A_{i}^{n^{n}}}}\left(t_{i}^{n}\right)^{p}\left(\frac{|P|}{\left|A_{i}^{n}\right|}\right)^{1-s p}\right]+2^{p}\left[\sum_{\substack{P \in \mathcal{Y}^{k}\left(P^{n}\right) \\
P \subset B_{i}^{n^{n}}}}\left(z_{i}^{n}\right)^{p}\left(\frac{|P|}{\left|B_{i}^{n}\right|}\right)^{1-s p}\right] \\
\leq & C_{54} \lambda_{6}^{\left(k-j_{0}^{*}\left(P_{i}^{n}\right)\right)(1-s p) .}
\end{aligned}
$$

So by Proposition 8.1.A in [27] (take $\mathcal{G}=\mathcal{W}=\mathcal{P}_{\star}, A=B=0$ and $k_{i}=i$ ) we have that $f \in \mathcal{B}_{p, q}^{s}\left(\mathcal{P}_{\star}\right)$. In particular $f \in L^{t}$, for some $t>1$.

Step III. In particular $f \in L^{t}$, for some $t>1$. Since $\left\{\phi_{S_{i}^{n}}\right\}$ belongs to a unconditional basis of $L^{t}$ we can write

$$
f=\sum_{j} \sum_{j_{0}^{\circ}\left(\hat{Q}_{i}^{n}\right)=j} \frac{1}{2^{n} i^{1 / p}\left|\hat{Q}_{i}^{n}\right|^{1 / p-s-1 / 2}} \phi_{S_{i}^{n}}
$$

Since $v_{n+1}>v_{n}+3 C_{46}+2, v_{n} \leq j_{0}^{\circ}\left(P_{i}^{n}\right) \leq v_{n}+C_{46}$ and $\hat{Q}_{i}^{n} \neq \hat{Q}_{\ell}^{n}$ for $i \neq \ell$ we conclude that

$$
\#\left\{\hat{Q}_{i}^{n}: v_{n} \leq j_{0}^{\circ}\left(\hat{Q}_{i}^{n}\right) \leq v_{n}+C_{46}\right\}=r_{n},
$$

so there is at least one $j \in\left[v_{n}, v_{n}+C_{46}\right]$ such that

$$
\sum_{\hat{Q}_{i}^{n} \in \mathcal{P}_{0}^{j}} \frac{1}{i} \geq \frac{2^{n p}}{C_{46}+1} \text {. }
$$

We conclude that

$$
\begin{aligned}
N_{\text {haar }}^{\circ}(f) & =\left(\sum_{j \geq 1}\left(\sum_{\hat{Q}_{i}^{n} \in \mathcal{P}_{\circ}^{j}} \frac{1}{2^{n p} i}\right)^{q / p}\right)^{1 / q} \\
& =\left(\sum_{n} \frac{1}{2^{n q}} \sum_{v_{n} \leq j \leq v_{n}+C_{46}}\left(\sum_{\hat{Q}_{i}^{n} \in \mathcal{P}_{\circ}^{j}} \frac{1}{i}\right)^{q / p}\right)^{1 / q} \\
& =\left(\sum_{n} \frac{1}{2^{n q}} \sum_{v_{n} \leq j \leq v_{n}+C_{46}}\left(\sum_{\hat{Q}_{i}^{n} \in \mathcal{P}_{\circ}^{j}} \frac{1}{i}\right)^{q / p}\right)^{1 / q}=\infty
\end{aligned}
$$

so $f \notin \mathcal{B}_{p, q}^{s}\left(\mathcal{P}_{\circ}\right)$.

This completes the proof of Claim 6 .

To complete the proof of Proposition 7.1, note that Claim 4. tell us that $C \Rightarrow A$ and $C \Rightarrow B$. Suppose that $A$ holds, that is $\mathcal{B}_{p, q}^{s}\left(\mathcal{P}_{\circ}\right) \subset \mathcal{B}_{p, q}^{s}\left(\mathcal{P}_{\star}\right)$. If $q<p$ then Claim 5. implies that (7.42) holds. So by Claim 3. we have that $h_{\circ}^{-1} \circ h_{\star}$ is a 
bi-Lipchitz function and Claim 4. gives $\mathcal{B}_{p, q}^{s}\left(\mathcal{P}_{\circ}\right)=\mathcal{B}_{p, q}^{s}\left(\mathcal{P}_{\star}\right)$. On the other hand if $p<q$ then Claim 6 (exchanging the roles of $\mathcal{P}_{\circ}$ and $\mathcal{P}_{\star}$ ) implies that

$$
\sup _{k} \#\left\{j_{0}^{\circ}(Q), Q \in \mathcal{P}_{\star}^{k}\right\}<+\infty
$$

so again by Claim 3. we have that $h_{\circ}^{-1} \circ h_{\star}$ is a bi-Lipchitz function and Claim 4. gives $\mathcal{B}_{p, q}^{s}\left(\mathcal{P}_{\circ}\right)=\mathcal{B}_{p, q}^{s}\left(\mathcal{P}_{\star}\right)$. We conclude that $A \Rightarrow B$ and $A \Rightarrow C$. Obviously $B \Rightarrow A$, so this concludes the proof.

Proposition 7.2 (Exotic Besov-ish spaces). Let $\mathbb{B}_{p, q}^{s}$ be the Besov space of $[0,1]$ as defined in Section 2. There are quasisymmetric grids $\mathcal{P}_{\star}$ such that $\mathcal{B}_{p, q}^{s}\left(\mathcal{P}_{\star}\right) \neq \mathbb{B}_{p, q}^{s}$.

Proof. Let $\mathbb{S}^{1}=\mathbb{R} / \mathbb{Z}$ and consider the smooth expanding map $f: \mathbb{S}^{1} \rightarrow \mathbb{S}^{1}$ given by $f(x)=2 x \bmod 1$. Note that the dyadic grid on $[0,1]$ is a sequence of Markov partitions for $f$, that is, $f: P \rightarrow f(P)$ is a difeomorphism for every $P \in \mathcal{D}^{k}$, with $k \geq 1$, and $f(P) \in \mathcal{D}^{k-1}$. Let $g: \mathbb{S}^{1} \rightarrow \mathbb{S}^{1}$ be a 2 to $1 C^{2}$-covering of the circle such that $g(0)=0$. Then there is a quasisymmetric homeomorphism $h_{\star}: \mathcal{S}^{1} \rightarrow \mathbb{S}^{1}$ such that $h_{\star}(0)=0$ and conjugates $f$ and $g$, that is, and $h_{\star} \circ g=f \circ h_{\star}$ on $\mathbb{S}^{1}$. In particular there is a quasisymmetric grid $\mathcal{P}_{\star}$ such that $h_{\star}\left(\mathcal{P}_{\star}\right)=\mathcal{D}$. We can choose $g$ in such way that for some $n \geq 1$ there is some $n$-periodic point $p$ of $g\left(g^{n}(p)=p\right)$ satisfying

$$
D g^{n}(p) \neq D f^{n}\left(h_{\star}(p)\right)=2^{n} .
$$

We claim that $h_{\star}$ is not a bi-Lipchitz map. Indeed, otherwise the conjugacy $h_{\star}$ would be absolutely continuous with respect to the Lebesgue measure. But Shub and Sullivan [26] proved that every absolutely continuous conjugacy between two $C^{2}$ expanding maps on the circle is indeed a $C^{1}$ difeomorphism. But this is not possible since in this case it is easy to show that $D g^{n}(p)=D f^{n}\left(h_{\star}(p)\right)$ for every $n$ periodic point $p$ of $g$. Since $h_{\star}$ is not a bi-Lipchitz map, Proposition 7.1 (take $h_{\circ}=I d$ and $\left.\mathcal{P}_{\circ}=\mathcal{D}\right)$ implies that $\mathcal{B}_{p, q}^{s}\left(\mathcal{P}_{\star}\right) \neq \mathcal{B}_{p, q}^{s}(\mathcal{D})=\mathbb{B}_{p, q}^{s}$.

Remark 7.3. One can ask if $\mathcal{B}_{p, q}^{s}\left(\mathcal{P}_{\star}\right)$ above is the Besov space of a homogenous space of the form $([0,1], \rho, m)$, where $m$ is the Lebesgue measure and $\rho$ is some metric on $[0,1]$ other than the euclidean one. We do not have a complete answer for that. However note that $\rho$ can not be the metric defined in (3.32). Indeed, if we consider a recalibration $\mathcal{G}$ of $\mathcal{P}_{\star}$ then we have that $\mathcal{G}$ is a good grid of intervals where every interval in the same level as more of less the same length. Using the same argument of the proof of Theorem 3.2 we conclude that $\mathcal{B}_{p, q}^{s}(\mathcal{G})$ is the Besov space $\mathbb{B}_{p, q}^{s}([0,1], d, m)=\mathcal{B}_{p, q}^{s}(\mathcal{D})$, where $d$ is the euclidean distance.

\section{GOOD GRID INVARIANCE FOR $\mathcal{B}_{p, p}^{s}$}

The next result tell us that when $p=q$ the Besov spaces $\mathcal{B}_{p, p}^{s}$ are far more resilient to modifications of the grid, what somehow remind us of Theorem 2.2 in Vodop/yanov [34]. See also Koch, Koskela, Saksman and Soto [18].

Proposition 8.1. Consider the interval $I=[0,1]$ with the Lebesgue measure $m$. Let $\mathcal{P}_{\circ}, \mathcal{P}_{\star}$ be grids of $(I, m)$ such that
A. We have that $\mathcal{P}_{0}^{k}, \mathcal{P}_{\star}^{k}$ are families of intervals for every $k$,
B. For every $k \neq j$ we have $\mathcal{P}_{\circ}^{k} \cap \mathcal{P}_{\circ}^{j}=\emptyset$ and
C. We have that $\mathcal{P}_{\star}$ is a $\left(\lambda_{5}, \lambda_{6}\right)$-good grid. 
Then

$$
\mathcal{B}_{p, p}^{s}\left(\mathcal{P}_{\circ}\right) \subset \mathcal{B}_{p, p}^{s}\left(\mathcal{P}_{\star}\right)
$$

and the inclusion is continuous. As a consequence $\mathcal{B}_{p, p}^{s}\left(\mathcal{P}_{\star}\right)=B_{p, p}^{s}$, where $B_{p, p}^{s}$ is the Besov space in the homogeneous space $(I, m)$.

Proof. We will prove it in three steps.

Step I. Note that given two grids $\mathcal{G}_{1}$ and $\mathcal{G}_{2}$ such that

$$
\mathcal{G}_{i}^{k} \cap \mathcal{G}_{i}^{j}=\emptyset
$$

for every $k \neq j, i=1,2$, and

$$
\bigcup_{k} \mathcal{G}_{1}^{k}=\bigcup_{k} \mathcal{G}_{2}^{k}
$$

then $\mathcal{B}_{p, p}^{s}\left(\mathcal{G}_{1}\right)=\mathcal{B}_{p, p}^{s}\left(\mathcal{G}_{2}\right)$ and the corresponding norms are equivalent.

Define $\mathcal{G}_{i}=\left(\mathcal{G}_{i}^{k}\right)_{k}$, with $i=\star, \circ$, in the following way.

$$
\mathcal{G}_{i}^{k}=\left\{P \in \bigcup_{j} \mathcal{P}_{i}^{j} \text { s.t. } \frac{1}{2^{k+1}}<|P| \leq \frac{1}{2^{k}}\right\} .
$$

Then $\mathcal{G}_{i}$ is a grid and consequently $\mathcal{B}_{p, p}^{s}\left(\mathcal{G}_{i}\right)=\mathcal{B}_{p, p}^{s}\left(\mathcal{P}_{i}\right)$ and the corresponding norms are equivalent. It remains to show the (continuous) inclusion $\mathcal{B}_{p, p}^{s}\left(\mathcal{G}_{\circ}\right) \subset \mathcal{B}_{p, p}^{s}\left(\mathcal{G}_{\star}\right)$.

Step II. Since $\mathcal{P}_{\star}$ is a good grid there is $r \geq 1$ such that for every $j \in \mathbb{N}$ and $x \in I$ there is $P \in \mathcal{G}_{\star}^{i}$, with $i \in[j+1, j+r]$, satisfying $x \in P$.

Step III. Given $Q \in \mathcal{G}_{\circ}^{k_{0}}$, define

$$
j_{0}=\min \left\{j \in \mathbb{N} \text {, there is } P \in \mathcal{G}_{\star}^{j} \text { satisfying } P \subset Q\right\} .
$$

By Step II we have that $k_{0}+r+2 \leq j_{0} \leq k_{0}$. Let

$$
\tilde{\mathcal{F}}^{j_{0}}(Q)=\left\{P \in \mathcal{G}_{\star}^{j_{0}}, \text { int } P \subset Q\right\}
$$

and

$$
A_{j_{0}}=\bigcup_{P \in \tilde{\mathcal{F}}^{j_{0}}(Q)} \bar{P}
$$

and by induction define

$$
\tilde{\mathcal{F}}^{j}(Q)=\left\{P \in \mathcal{G}_{\star}^{j}, \text { int } P \subset Q \backslash \cup_{i<j} A_{i}\right\} .
$$

and

$$
A_{j}=\bigcup_{P \in \tilde{\mathcal{F}}^{j}(Q)} \bar{P}
$$

Since $\mathcal{P}_{\star}$ is a nested sequence of partitions we can find a subfamily $\mathcal{F}^{j}(Q) \subset \tilde{\mathcal{F}}^{j}(Q)$ such that

$$
A_{j}=\bigcup_{P \in \mathcal{F}^{j}(Q)} \bar{P}
$$

and $\mathcal{F}^{j}(Q)$ is a family of pairwise disjoint intervals. By definition $\left\{A_{j}\right\}_{i}$ is a family of sets with pairwise disjoint interior. Note that

$$
D_{j}=\cup_{i \leq j} A_{i} .
$$


can be described as

$D_{j}=\left\{x \in Q\right.$ s.t. there is $P \in \mathcal{G}_{\star}^{i}$, with $i \leq j$, such that $\left.x \in P \subset Q\right\}$.

Let $\left[a_{j}, b_{j}\right]$ be the convex hull of $D_{j}$. If $x \in\left[a_{j}, b_{j}\right] \backslash D_{j}$ then

$$
\operatorname{dist}\left(x,\left\{a_{j}, b_{j}\right\}\right)>\frac{1}{2^{j}},
$$

so by Step II there is $P \in \mathcal{G}_{\star}^{i}$, with $i \in[j+1, j+r]$, such that $x \in P \subset\left[a_{j}, b_{j}\right] \subset Q$. Note that $P \cap W=\emptyset$ for every $W \in \tilde{\mathcal{F}}^{j}(Q)$, otherwise we would have $x \in P \subset$ $W \subset A_{j}$. In particular $x \in \mathcal{F}^{i}(Q)$. Consequently

$$
\left[a_{j}, b_{j}\right] \subset D_{j+r}=\cup_{i \leq j+r} A_{i} .
$$

Additionally if $y \in\left\{a_{j}, b_{j}\right\}$ satisfies

$$
\operatorname{dist}(y, \partial Q)>\frac{1}{2^{j-r}}
$$

then by Step III there is $P \in \mathcal{G}_{\star}^{i}$, with $i \in[j-r+1, j]$, such that $P \subset Q$ and $P \cap\left(\left[0, a_{j}[\cup] b_{j}, 1\right]\right) \neq \emptyset$, which contradicts the inclusion $D_{j} \subset\left[a_{j}, b_{j}\right]$. We conclude that

SO

$$
m\left(Q \backslash D_{j+r}\right)<\frac{1}{2^{j-r-1}},
$$

$$
\# \mathcal{F}^{j+r+1}(Q) \leq 2^{j+r+2} m\left(Q \backslash D_{j+r}\right) \leq 2^{2 r+1} .
$$

and for every $\ell$ satisfying $j_{0} \leq \ell \leq j_{0}+r$ we have

$$
\# \mathcal{F}^{\ell}(Q) \leq 2^{\ell+1} m(Q) \leq 2^{r+1},
$$

so $\# \mathcal{F}^{\ell}(Q) \leq 2^{2 r+1}$ for every $\ell \geq j_{0}$. If $a_{W}$ is the canonical Souza's atom on $W$ and $Q \in \mathcal{G}_{\circ}^{k_{0}}$ we have

$$
a_{Q}=\sum_{j \geq j_{0}} \sum_{P \in \mathcal{F}^{j}(Q)}\left(\frac{|P|}{|Q|}\right)^{1 / p-s} a_{P}
$$

and for every $j \geq j_{0}$

$$
\sum_{P \in \mathcal{F}^{j}(Q)}\left(\frac{|P|}{|Q|}\right)^{1-s p} \leq 2^{2 r+1}\left(\frac{2^{-j}}{2^{-\left(k_{0}+1\right)}}\right)^{1-s p} \leq 2^{2 r+1-(r+2)(1-s p)} 2^{-\left(j-j_{0}\right)(1-s p)}
$$

By Propositon 8.1.C in [27] we have that $\mathcal{B}_{p, p}^{s}\left(\mathcal{G}_{\circ}\right) \subset \mathcal{B}_{p, p}^{s}\left(\mathcal{G}_{\star}\right)$ and moreover this inclusion is continuous. In particular $\mathcal{B}_{p, p}^{s}\left(\mathcal{G}_{\star}\right)=\mathcal{B}_{p, p}^{s}(\mathcal{D})$, and by Propostition 2.5 we have $\mathcal{B}_{p, p}^{s}(\mathcal{D})=B_{p, p}^{s}$.

\section{REFERENCES}

[1] H. Aimar, B. Iaffei, and L. Nitti. On the Macías-Segovia metrization of quasi-metric spaces. Rev. Un. Mat. Argentina, 41(2):67-75, 1998.

[2] R. Alvarado and M. Mitrea. Hardy spaces on Ahlfors-regular quasi metric spaces, volume 2142 of Lecture Notes in Mathematics. Springer, Cham, 2015. A sharp theory.

[3] A. Arbieto and D. Smania. Transfer operators and atomic decomposition. arXiv 1903.06943, 2019.

[4] O. V. Besov. On some families of functional spaces. Imbedding and extension theorems. Dokl. Akad. Nauk SSSR, 126:1163-1165, 1959.

[5] G. Bourdaud. Changes of variable in Besov spaces. II. Forum Math., 12(5):545-563, 2000.

[6] G. Bourdaud and W. Sickel. Changes of variable in Besov spaces. Math. Nachr., 198:19-39, 1999. 
[7] M. Christ. A $T(b)$ theorem with remarks on analytic capacity and the Cauchy integral. Colloq. Math., 60/61(2):601-628, 1990.

[8] R. R. Coifman and G. Weiss. Analyse harmonique non-commutative sur certains espaces homogènes. Lecture Notes in Mathematics, Vol. 242. Springer-Verlag, Berlin-New York, 1971. Étude de certaines intégrales singulières.

[9] G. S. de Souza. The atomic decomposition of Besov-Bergman-Lipschitz spaces. Proc. Amer. Math. Soc., 94(4):682-686, 1985.

[10] G. S. de Souza. Two more characterizations of Besov-Bergman-Lipschitz spaces. Real Anal. Exchange, 11(1):75-79, 1985/86. The ninth summer real analysis symposium (Louisville, Ky., 1985).

[11] G. S. de Souza, R. O'Neil, and G. Sampson. Several characterizations for the special atom spaces with applications. Rev. Mat. Iberoamericana, 2(3):333-355, 1986.

[12] R. A. DeVore and V. A. Popov. Interpolation of Besov spaces. Trans. Amer. Math. Soc., 305(1):397-414, 1988.

[13] M. Frazier and B. Jawerth. Decomposition of Besov spaces. Indiana Univ. Math. J., 34(4):777-799, 1985.

[14] C. Gu and M. Taibleson. Besov spaces on nonhomogeneous martingales. In Harmonic analysis and discrete potential theory (Frascati, 1991), pages 69-84. Plenum, New York, 1992.

[15] Y. Han, S. Lu, and D. Yang. Inhomogeneous Besov and Triebel-Lizorkin spaces on spaces of homogeneous type. Approx. Theory Appl. (N.S.), 15(3):37-65, 1999.

[16] Y. S. Han and E. T. Sawyer. Littlewood-Paley theory on spaces of homogeneous type and the classical function spaces. Mem. Amer. Math. Soc., 110(530):vi+126, 1994.

[17] T. Hytönen and A. Kairema. Systems of dyadic cubes in a doubling metric space. Colloq. Math., 126(1):1-33, 2012.

[18] H. Koch, P. Koskela, E. Saksman, and T. Soto. Bounded compositions on scaling invariant Besov spaces. J. Funct. Anal., 266(5):2765-2788, 2014.

[19] P. Koskela, D. Yang, and Y. Zhou. Pointwise characterizations of Besov and Triebel-Lizorkin spaces and quasiconformal mappings. Adv. Math., 226(4):3579-3621, 2011.

[20] R. A. Macías and C. Segovia. Lipschitz functions on spaces of homogeneous type. Adv. in Math., 33(3):257-270, 1979.

[21] P. Oswald. On function spaces related to finite element approximation theory. Z. Anal. Anwendungen, 9(1):43-64, 1990.

[22] P. Oswald. Multilevel finite element approximation. Teubner Skripten zur Numerik. [Teubner Scripts on Numerical Mathematics]. B. G. Teubner, Stuttgart, 1994. Theory and applications.

[23] M. Paluszyński and K. Stempak. On quasi-metric and metric spaces. Proc. Amer. Math. Soc., 137(12):4307-4312, 2009.

[24] J. Peetre. New thoughts on Besov spaces. Mathematics Department, Duke University, Durham, N.C., 1976. Duke University Mathematics Series, No. 1.

[25] F. Przytycki and M. Urbański. Conformal fractals: ergodic theory methods, volume 371 of London Mathematical Society Lecture Note Series. Cambridge University Press, Cambridge, 2010.

[26] M. Shub and D. Sullivan. Expanding endomorphisms of the circle revisited. Ergodic Theory Dynam. Systems, 5(2):285-289, 1985.

[27] D. Smania. Besov-ish spaces through atomic decomposition. arXiv 1903.06937, 2019.

[28] E. M. Stein. Singular integrals and differentiability properties of functions. Princeton Mathematical Series, No. 30. Princeton University Press, Princeton, N.J., 1970.

[29] H. Triebel. Non-smooth atoms and pointwise multipliers in function spaces. Ann. Mat. Pura Appl. (4), 182(4):457-486, 2003.

[30] H. Triebel. A new approach to function spaces on quasi-metric spaces. Rev. Mat. Complut., 18(1):7-48, 2005.

[31] H. Triebel. Theory of function spaces. Modern Birkhäuser Classics. Birkhäuser/Springer Basel AG, Basel, 2010. Reprint of 1983 edition [MR0730762], Also published in 1983 by Birkhäuser Verlag [MR0781540].

[32] H. Triebel. Fractals and spectra. Modern Birkhäuser Classics. Birkhäuser Verlag, Basel, 2011. Related to Fourier analysis and function spaces.

[33] M. Urbański. Measures and dimensions in conformal dynamics. Bull. Amer. Math. Soc. (N.S.), 40(3):281-321, 2003. 
[34] S. K. Vodop/yanov. Mappings of homogeneous groups and embeddings of function spaces. Sibirsk. Mat. Zh., 30(5):25-41, 215, 1989.

[35] D. Yang. Besov spaces on spaces of homogeneous type and fractals. Studia Math., 156(1):1530,2003

Departamento de Matemática, Intituto de Ciências Matemáticas e da ComputaçãoUniversidade de São Paulo (ICMC/USP) - São Carlos, Caixa Postal 668, São Carlos-SP, CEP 13560-970, BRAZIL.

E-mail address: smania@icmc.usp.br

URL: http://conteudo.icmc.usp.br/pessoas/smania/ 